\title{
Superconductivity and electrical resistivity in alkali metal doped fullerides: Phonon mechanism
}

\author{
DINESH VARSHNEY*, A DUBE, K K CHOUDHARY ${ }^{\dagger}$ and R K SINGH ${ }^{\ddagger}$ \\ School of Physics, Vigyan Bhawan, Devi Ahilya University, Khandwa Road Campus, Indore 452 017, India \\ ${ }^{\dagger}$ Department of Physics, SVITS, Indore 453 331, India \\ Department of Physics, Barkatullah University, Bhopal 462 026, India
}

MS received 22 September 2004

\begin{abstract}
We consider a two-peak model for the phonon density of states to investigate the nature of electron pairing mechanism for superconducting state in fullerides. We first study the intercage interactions between the adjacent $\mathrm{C}_{60}$ cages and expansion of lattice due to the intercalation of alkali atoms based on the spring model to estimate phonon frequencies from the dynamical matrix for the intermolecular alkali- $\mathrm{C}_{60}$ phonons. Electronic parameter as repulsive parameter and the attractive coupling strength are obtained within the random phase approximation. Transition temperature, $T_{c}$, is obtained in a situation when the free electrons in lowest molecular orbital are coupled with alkali- $\mathrm{C}_{60}$ phonons as $5 \mathrm{~K}$, which is much lower as compared to reported $T_{\mathrm{c}}(\approx 20 \mathrm{~K})$. The superconducting pairing is mainly driven by the high frequency intramolecular phonons and their effects enhance it to $22 \mathrm{~K}$. To illustrate the usefulness of the above approach, the carbon isotope exponent and the pressure effect are also estimated. Temperature dependence of electrical resistivity is then analysed within the same model phonon spectrum. It is inferred from the two-peak model for phonon density of states that high frequency intramolecular phonon modes play a major role in pairing mechanism with possibly some contribution from alkali- $\mathrm{C}_{60}$ phonon to describe most of the superconducting and normal state properties of doped fullerides.
\end{abstract}

Keywords. Fullerenes; alkali- $\mathrm{C}_{60}$ phonon; on-ball- $\mathrm{C}_{60}$ phonon; pressure effect; electrical resistivity.

\section{Introduction}

Buckminsterfullerenes are known to make compounds with alkali metals intensively studied mainly due to superconductivity and the variation of compounds (Hebard et al 1991; Holczer et al 1991; Tanigaki et al 1991). The doping in $\mathrm{C}_{60}$ as $\mathrm{M}_{x} \mathrm{C}_{60}(\mathrm{M}=\mathrm{K}, \mathrm{Rb}, \mathrm{Cs} ; x=1,2$ and 3) and $\left(\mathrm{NH}_{3}\right) \mathrm{K}_{3} \mathrm{C}_{60}$ is the subject of renewed interest because of its technological and industrial importance. In close relation the nature of attractive pairing mechanism with conventional phonon mediated electron pairing or unconventional electronic mechanisms in fullerides $\left(T_{\mathrm{c}} \sim\right.$ $20-45 \mathrm{~K}$ ) remains a central point of several experimental studies. The normal state electronic and magnetic properties of alkali metal intercalated fullerides are reviewed at great length (Dresselhaus et al 1996; Gunnarson 1997; Forro and Mihaly 2001).

Neutron inelastic scattering measurement (Prassides et al 1991; Pintschovius 1996) probes that the vibrational spectrum may be conveniently divided into two regions. One of them belongs to the rotation of $\mathrm{C}_{60}$ molecule and the intermolecular vibrations $(\sim 2 \cdot 5-25 \mathrm{meV})$ and the others are the intramolecular mode with frequency, $25-200 \mathrm{meV}$. Raman scattering (Mitch et al 1992) yields the on-ball

*Author for correspondence (vdinesh33@ rediffmail.com)
$\mathrm{C}_{60}$ vibrational modes ( $\left.40 \mathrm{meV}-0 \cdot 2 \mathrm{eV}\right)$. Lattice dynamical studies (Belosludov and Shpakov 1991) point out that the spectrum consists of mode of $\mathrm{C}_{60}\left(26 \mathrm{~cm}^{-1}\right)$, the vibrations of $\mathrm{K}^{+}$ions, the translational vibrations as a whole $\left(\sim 45-120 \mathrm{~cm}^{-1}\right)$ and the $\mathrm{C}_{60}$ vibrations ( 260$\left.1900 \mathrm{~cm}^{-1}\right)$. The wide frequency range of phonon spectrum, however, raises an important issue to clarify which of these molecular phonon modes induce the superconductivity in doped fullerides.

The energy scales of the various phonon modes that mediate the electron-phonon coupling are different, thanks to the dependence of transition temperature on isotopic mass, a good probe to clarify phonon mechanism. The carbon isotope effect demonstrates $\alpha_{c}=0.30 \pm 0.05$ with $100 \%$ substitution of ${ }^{6} \mathrm{C}_{13}$ for ${ }^{6} \mathrm{C}_{12}$ (Chen and Lieber 1993) and naturally favours the phonon mediated pairing and ruled out electronic in origin. Fuhrer and coworkers (1991) documented a reduced $\alpha_{c}$ of about $0 \cdot 21 \pm 0 \cdot 012$. It is apparent that conventional BCS weak coupling theory predicts one half value of isotope effect and reduced value of $\alpha_{c}$ than BCS result is quite intriguing.

It is worth to point out that it is the alkali metal isotope effect and not the carbon isotope effect which will identify whether inter or intramolecular phonon modes induce the superconductivity. Burk and coworkers (1994) have shown a $\mathrm{Rb}$ isotope effect of $\alpha_{\mathrm{Rb}}=-0.028 \pm 0.036$, a result which implies that the alkali- $\mathrm{C}_{60}$ optic phonons play at 
most a minor role in the pairing mechanism. The small negative $\alpha_{\mathrm{Rb}}$ could possibly be an artifact anharmonic alkali dopant potential in the interstitial sites. Although reduced value of $\alpha_{c}$ imposes a constraint on the conventional phonon pairing in fullerides, but also raises the possibility of alkali- $\mathrm{C}_{60}$ optic phonons if they contribute to pairing, whose alkali-metal isotope effect exponent may be masked by the effects of an anharmonic potential.

Tunneling measurements predict a large energy gap ratio $\left(\beta=2 \Delta_{0} / k_{\mathrm{B}} T_{\mathrm{c}}\right)$ of $5 \cdot 3 \pm 0 \cdot 2$ for $\mathrm{K}_{3} \mathrm{C}_{60}$ which leads to strong coupling regime (Zhang et al 1991b). Although tunneling measurements predict a large magnitude of the reduced energy gap then the BCS value of 3.53 supports a low energy intermolecular phonon as the source of superconductivity. Other techniques as infrared spectroscopy (DeGiorgi et al 1994), muon spin relaxation measurement ( $\mu$ SR) (Kiefl et al 1993), reflectivity measurement (DeGiorgi et al 1992) and nuclear spin relaxation measurement (Tycko et al 1992) yield a energy gap value of $3 \cdot 6 \sim 4 \cdot 0$ close to the BCS weak coupling limit. The spin relaxation measurement suggests that the coupling between the electrons and the intermolecular phonons or radial intramolecular phonons is important for superconductivity in $\mathrm{K}_{3} \mathrm{C}_{60}$. Optical studies (Koller et al 1996) on $\mathrm{Rb}_{3} \mathrm{C}_{60}$ led to $\beta=4.2$ which indicates that the superconductivity cannot be described in the weak coupling limit. Hence, these measurements (Zhang et al 1991b; DeGiorgi et al 1992, 1994; Tycko et al 1992; Kiefl et al 1993; Koller et al 1996) although favour the electron-phonon coupling but questioned whether the weak or strong coupling limit applies.

Mechanisms for pairing in fullerides based on either intramolecular or with intermolecular phonons have been suggested. Zhang and coworkers (1991a) first proposed that the doped alkali- $\mathrm{C}_{60}$ phonon mode produces a strong attraction for electrons on $\mathrm{C}_{60}$ using a Hubbard model to describe the superconductivity. Varma et al (1991) stress for the fact that the electron-phonon interaction in fulleride be induced by high frequency intramolecular vibrational modes on a ball of $\mathrm{C}_{60}$ and it should be a key feature of superconductivity. The same follows from Schluter et al (1992). Kresin (1992) stressed that the strong electron-intramolecular phonon coupling will describe the superconducting state.

On the other hand, Zhang and Guo (1994) stressed that the coupled $\mathrm{K}^{+}$optical mode and the vibration of $\mathrm{C}_{60}$ mass centre will induce a strong electron-phonon interaction. A three square well model which is characterized by windows of low-frequency intermolecular phonons, high frequency intramolecular and Coulomb energy allows a coherent interpretation of superconducting fulleride properties (Ivanov and Maruyama 1995). Furthermore, Alexandrov and Kabanov (1996) formulated a non-adiabatic theory of superconductivity taking into account the polaron band narrowing and the realistic electron-phonon as well as the Coulomb interactions. By and large these approaches favour the participation of on ball $\mathrm{C}_{60}$ intramolecular phonon for superconducting state with an experimental support of small negative $\alpha_{\mathrm{Rb}}$ that rules out the possible participation of intermolecular phonons.

Study on transition temperature under high pressure is another convincing test for determination of the participation of the exact phonon mode in the wide energy scale of phonon spectrum. In the doped fullerides, $T_{\mathrm{c}}$ monotonically changes with the unit cell size (lattice constant), $a$. The pressure effect in fullerides is huge, $\mathrm{d} T_{\mathrm{c}} / \mathrm{d} P$ is negative, $\mathrm{d} T_{\mathrm{c}} / \mathrm{d} \ln a$ for different fullerides are close to each other. Sparn and coworkers (1992) noticed that for $\mathrm{K}_{3} \mathrm{C}_{60}$, $T_{\mathrm{c}}(=19 \cdot 3 \mathrm{~K})$, decreases strongly with increasing pressure, $\mathrm{d} T_{\mathrm{c}} / \mathrm{d} P=-7.8 \mathrm{~K} / \mathrm{GPa}$. Subsequently, measuring magnetic susceptibility (Diederich et al 1996) under hydrostatic pressure yields $\mathrm{d} T_{\mathrm{c}} / \mathrm{d} P=-9.7 \mathrm{~K} / \mathrm{GPa}$ with a $T_{\mathrm{c}}$ of $29.6 \mathrm{~K}$ for single-phase $\mathrm{Rb}_{3} \mathrm{C}_{60}$. Chaban (2002) argued that the dependence of $T_{\mathrm{c}}$ on pressure is to be connected with the chemical pressure effect in fullerides.

In this respect, it is interesting to look at the nature of superconducting state, to which the energy scale of the mediating boson in the electron-pairing interaction is crucial in determining whether weak or strong coupling is appropriate. These experimental and theoretical understanding on fullerides motivates us to develop a theory with both inter- and intramolecular phonon to reveal the attractive pairing mechanism as well as the understanding of the transition temperature and its pressure (volume) dependence of fullerides. The idea borne in mind is mainly due to two-fold reasons. Firstly the intermolecular vibrations possess energy in the range $2 \cdot 5-25 \mathrm{meV}$ and the band structure calculations (Erwin and Pickett 1992; Satapthy et al 1992) reveal the Fermi energy $\left(\varepsilon_{\mathrm{F}}\right)$ as $0.2 \sim 0.3 \mathrm{eV}$. Thus, fullerides obey $\omega_{\mathrm{er}}<\varepsilon_{\mathrm{F}}$, so the consideration of intermolecular vibrations, i.e. the coupling of the conduction electrons with the intermolecular phonons, will be appropriate. Secondly, the coupling with the displacements of the alkali- $\mathrm{C}_{60}$ optic is definitely important to the electron-phonon interaction, because of finite positive $\alpha_{c}$ in $\mathrm{K}_{3} \mathrm{C}_{60}$.

To be complete, the normal state resistivity measurements on doped fullerides reveal its metallic behaviour. Xiang and coworkers (1992) have first documented the resistivity measurement data of single crystal $\mathrm{K}_{3} \mathrm{C}_{60}$. The observed temperature dependence can be accounted for with an electron-phonon scattering mechanism if there is a high frequency contribution from the intraball phonons and a lower frequency contribution from phonons. The overall temperature dependence of $\rho(T)$ above $T_{\text {c }}$ places constraints on the normal state transport models. Palstra et al (1992) argued that $\mathrm{K}_{3} \mathrm{C}_{60}$ is near metal-insulator transition as the effective mean free path and interatomic distances are of similar order in thin films. A good fit to temperature dependent resistivity of $\mathrm{K}_{3} \mathrm{C}_{60}$ was obtained only by including a phonon mode with $\theta^{*}=185 \mathrm{~K}(\mathrm{Gel}-$ fand and Lu 1992). Crespi et al (1992) have analysed the 
single crystal $\mathrm{K}_{3} \mathrm{C}_{60}$ resistivity data using Ziman's resistivity formula and show that both high frequency intraball and low frequency interball modes will account for the reported behaviour of resistivity.

Although the low frequency interball phonons will be strongly temperature dependent, the fit to the Ziman formula suggests that the coupling to these modes is small (Varshney and Singh 1997; Varshney et al 1998). The normal state resistivity measurement (Crespi et al 1992; Gelfand and Lu 1992; Palstra et al 1992; Xiang et al 1992; Goldoni et al 2001) points to the fact that both on ball molecule vibrations and alkali $\mathrm{C}_{60}$ optic phonons in the metallic state are important in retrieving the measured data. However, it is unclear that the normal state resistivity minima will correspond to inter- or intra-molecular phonons. Henceforth, the second objective of the present study is to look for the fact that which phonon modes in particular best reproduce the form of temperature dependent resistivity apart from their major role in pairing mechanism.

The present investigations are organized as follows. In $\S 2$, we introduce the model and sketch the formalism applied. As a first step we derive the force constants and the longitudinal phonon frequency for $\mathrm{C}_{60}$ molecule. Furthermore, a relationship in between force constant for undoped and alkali metal doped fullerenes has been established. The longitudinal phonon modes of $\mathrm{K}_{3} \mathrm{C}_{60}$ propagating along 110 directions are evaluated based on the spring model. The effective coupling strength between the conduction is then deduced and renormalized Coulomb repulsive parameter for the intermolecular phonon frequency is used to estimate $T_{\mathrm{c}}$. Later on, the intramolecular phonons are used for the estimation of $T_{\mathrm{c}}$. Developing this scheme, we have estimated the carbon isotope effect, the energy gap parameter, the pressure effect and normal state resistivity for $\mathrm{K}_{3} \mathrm{C}_{60}$.

The details of numerical analysis are presented along with discussion in $\S 3$. The purpose of the present investigation is to improve the understanding of various physical properties by including the effects of Coulomb, inter- and intramolecular phonons. However, we do not claim the process to be rigorous, but the results we report here do indeed shed light on the nature of these interactions in the test material. Conclusions are discussed in $\$ 4$ where we provide physical descriptions of the model calculations herein.

\section{Formalism and model}

To begin with, we consider the undoped $\mathrm{C}_{60}$ molecule. It is a pure carbon compound and possesses a spherical structure. The nuclear cage of $\mathrm{C}_{60}$ (the $\mathrm{C}_{60}$ sphere) has a diameter of $7 \cdot 1 \AA$ with 2 bond lengths, (6-6 ring) $1.4 \AA$ and (6-5 ring) $1.46 \AA$. The distance of the nearest approach in the molecular solid is $3.1 \AA$ (Martins and Troullier 1992). The $\mathrm{C}_{60}$ molecule crystallizes in the $f c c$ phase with lattice parameter, $a=14.20 \AA$ and the distance between the centres of the nearest neighbour $\mathrm{C}_{60}$ cages is $b=a / \sqrt{ } 2$. Furthermore, in $\mathrm{C}_{60}$ molecule, out of 4 valence electrons on each carbon atom, 3 electrons participate in the $90 \sigma$ bonds along the edges of truncated icosahedron and the remaining 60 electrons of the molecule are in the orbitals. In fact, the diameter of $\mathrm{C}_{60}$ molecule is large in such a way that the electron density is localized near the surface of the sphere. Electronic energy band structure (Satapthy et al 1992) reveals that for $\mathrm{C}_{60}$, the lowest unoccupied molecular orbital is empty and can accommodate 6 electrons. The $\mathrm{C}_{60}$ cages may be regarded as rigid spheres and a bandwidth develops from the weak interactions between the nearest neighbour $(n-n) \mathrm{C}_{60}$ cages. We begin with the $n-n \mathrm{C}_{60}$ cages interactions of undoped $\mathrm{C}_{60}$ molecule and derive the force constants along with the phonon frequencies of the $f c c$ lattice.

\subsection{Dispersion relation of undoped $C_{60}$}

To begin with, we consider the undoped $\mathrm{C}_{60}$ molecule, as a cube with $N$ cages and volume is $N a^{3} / 4$. When the volume is compressed from $V$ to $V+\mathrm{d} V$ the work done is

$$
W=\frac{1}{2} B \frac{\mathrm{d} V}{V} \mathrm{~d} V=B \frac{(\mathrm{d} V)^{2}}{2 V},
$$

$B$ being the bulk modulus and $a$ the lattice parameter. For a cube $V=a^{3}$ and $\mathrm{d} V / V=3 \mathrm{~d} a / a$. Keeping in mind the contribution of $6 N$ bonds between $n-n \mathrm{C}_{60}$ cages, $W$ is expressed as

$$
W=(3 N / \kappa)[\mathrm{d} a / \sqrt{2}]^{2},
$$

with $\kappa$ being the force constant for $n-n$ cages. A comparison yields, $\kappa=3 \mathrm{Ba} / 4$.

A set of $\mathrm{C}_{60}$ molecule is treated as a three-dimensional monoatomic lattice with atomic mass and the position of cage is denoted as $m_{k}$ and $u\left(\begin{array}{l}l \\ k\end{array}\right)$, respectively. The lattice considered $S$ unit cells numbered by an index $l=1,2 \ldots$ $S$ and for masses $k(=1)$ (Maraduddin et al 1963). The equations of motion along 110-direction are obtained from force $-\partial \Phi / \partial u\left(\begin{array}{l}l \\ k\end{array}\right)$ acting on the atom $\left(\begin{array}{l}l \\ k\end{array}\right)$ with $\Phi$ as the potential energy of the crystal. Hence equation of motion reads

$$
-m_{k} \ddot{u}_{j}\left(\frac{l}{k}\right)=\kappa\left[2 u_{j}\left(\frac{l}{k}\right)-u_{j+1}\left(\frac{l}{k}\right)-u_{j-1}\left(\frac{l}{k}\right)\right],
$$

also

$$
-m \ddot{u}_{j}(l)=\kappa\left[2 u_{j}(l)-u_{j+1}(l)-u_{j-1}(l)\right] .
$$

We express the equilibrium position of the $l$ th unit cell relative to an origin located at some atom is $r(l)=l_{1} a+$ $l_{2} b+l_{3} c$, where $l_{1}, l_{2}, l_{3}$ are integers and $a, b, c$ are the 
primitive translational vectors. In the following the plane wave solution as $u_{j}(l)=A(q) \exp i\left[q_{j} r(l)-\omega t\right]$, with $A$ as the amplitude, $q$ the wave-vector in 110 direction is used and $\omega(q)$ the angular frequency. Hence, the dispersion relation reads

$$
\omega_{L}(q)=2 \sqrt{\kappa / m}=\sqrt{3 B a / m},
$$

at the zone boundary $(q=\pi / b)$. The above is the longitudinal phonon mode, which is due to the rotational motion of the $\mathrm{C}_{60}$ molecule. The lattice is expanded due to intercalation of alkali metal $(\mathrm{K}, \mathrm{Rb}$ and $\mathrm{Cs})$ at tetrahedral and octahedral sites when $\mathrm{C}_{60}$ molecule is doped. We discuss this in the following subsection.

\subsection{Dispersion relation of alkali metal intercalated $C_{60}$}

The $\mathrm{C}_{60}$ molecule when chemically substituted with alkali metal $(\mathrm{K})$ atoms, the semiconducting $\mathrm{C}_{60}$ becomes metallic, at the $\mathrm{K}_{3} \mathrm{C}_{60}$ composition and it shows the superconducting nature. With the doping, $\mathrm{K}$ atoms become fully ionized in the $\mathrm{C}_{60}$ crystal and give up their electrons to highly polarizable $\mathrm{C}_{60}$ molecule. These electrons go to de-localized lowest unoccupied molecular orbitals, which can accommodate six electrons. The conduction band is empty for $\mathrm{C}_{60}$ crystal and for $\mathrm{K}_{3} \mathrm{C}_{60}$ the conduction band is half filled up to the Fermi level. The alkali atoms, $\mathrm{K}$, were located in two non-equivalent tetrahedral and an octahedral position of the lattice. In doped fullerides, there exists $6 \mathrm{~N} \mathrm{C}_{60}-\mathrm{C}_{60}$ bonds at a bond distance of $a / \sqrt{ } 2$, also $8 \mathrm{~N} \mathrm{C}_{60}-\mathrm{K}$ bonds with bond distance of $a \sqrt{ } 3 / 4$ with $a=14.28 \AA$ for $\mathrm{K}_{3} \mathrm{C}_{60}$. In the true sense, a dopant entering into parent lattice $\mathrm{C}_{60}$ tends to expand the lattice for creating a place for itself.

The transfer of charge will certainly result into some contraction and the final lattice parameter of the doped material will be the resultant of these effects. It is noticed that the lattice parameter for $\mathrm{K}_{3} \mathrm{C}_{60}(a=14 \cdot 28 \AA), \mathrm{Rb}_{3} \mathrm{C}_{60}$ $(a=14.45 \AA)$ and $\mathrm{Cs}_{3} \mathrm{C}_{60}(a=14.60 \AA)$ are more than the lattice parameter of undoped $\mathrm{C}_{60}$ viz. $a=14.20 \AA$ (Hebard et al 1991) and hence there is a resultant expansion. Furthermore, out of $8 \mathrm{~N} \mathrm{C}_{60}-\mathrm{K}$ bonds $4 \mathrm{~N}$ are at octahedral position and $4 \mathrm{~N}$ takes the tetrahedral position. For the sake of simplicity, the $\mathrm{K}$ atoms are considered at the tetrahedral site, as the cavity is large enough to accommodate $\mathrm{K}$ atom. The total bond energy within the harmonic approximation is expressed as

$$
E_{\mathrm{B}}=6 N \frac{\kappa}{2}\left[\frac{a}{\sqrt{2}}-r_{\mathrm{cc}}\right]^{2}+8 N \frac{\kappa^{\prime}}{2}\left[\frac{a \sqrt{3}}{4}-r_{\mathrm{mc}}\right]^{2},
$$

where $r_{\mathrm{cc}}$ is the equilibrium $\mathrm{C}_{60}-\mathrm{C}_{60}$ bond distance $(10.04 \AA)$ and $r_{\mathrm{mc}}$ denotes the metal- $\mathrm{C}_{60}$ bond distance and is $r_{\mathrm{mc}}=\left(r_{\mathrm{cc}} / 2\right)+r_{i}$, with $r_{i}=1.63 \AA$ is the ionic radius of $\mathrm{K}$. A relationship in between the force constants $\kappa$ $\left(\mathrm{C}_{60}-\mathrm{C}_{60}\right)$ and $\kappa^{\prime}\left(\mathrm{M}-\mathrm{C}_{60}\right)$ is established when the total bond energy reaches its maximum at the experimental value of lattice parameter, $a$.

Let us now consider the $n-n$ interactions between the $\mathrm{C}_{60}$ cages and $\mathrm{C}_{60}$ cages are bonded to both $\mathrm{K}$ atoms as well as adjacent $\mathrm{C}_{60}$ cages while the $\mathrm{K}$ atoms are bonded to $\mathrm{C}_{60}$ cages only. Treating $\mathrm{K}_{3} \mathrm{C}_{60}$ as a three-dimensional (3D) diatomic lattice with atomic masses as $m(M)$ and positions $u(v)$ for $\mathrm{C}_{60}(\mathrm{~K})$, the equations of motion follows

$$
\begin{aligned}
-m \ddot{u}_{j}(l)= & \kappa\left[2 u_{j}(l)-u_{j+1}(l)-u_{j-1}(l)\right]+ \\
& 2 \kappa_{1}\left[2 u_{j}(l)-v_{j+1 / 2}(l)-v_{j-1 / 2}(l)\right], \\
-M \ddot{v}_{j}(l)= & \kappa_{1}\left[2 v_{j}(l)-u_{j+1 / 2}(l)-u_{j-1 / 2}(l)\right] .
\end{aligned}
$$

We employ the plane wave solutions as $u_{j}(l)=A \exp$ $i\left[q_{j} r(l)-\omega t\right]$ and $v_{j}(l)=B \exp i\left[q_{j} r(l)-\omega t\right]$. The relation $\kappa_{1}$ is $0.66 \kappa^{\prime}$. It is useful to expand the coefficient $\Phi$, and the atomic force constants about its equilibrium value using the 3D forms of Taylor theorem for small displacements. Following harmonic approximation, all cubic and higher order terms are neglected and also the nearest neighbour interactions naturally demand the force constants and several other parameters in 3D (Maraduddin et al 1963). For various symmetries in $\mathrm{K}_{3} \mathrm{C}_{60}$, several transverse and longitudinal frequencies will be then obtained from the 3D dynamical matrix and are rigorous which indeed is not the scope of the present analysis. Confining only for the longitudinal phonon modes along 110-direction, the dispersion relation reads for small $q$ value

$$
\begin{aligned}
& \omega_{+}^{2}=4\left[D_{1}+D_{2}\right]-D_{1} D_{2}\left[D_{1}+D_{2}\right]^{-1}(q b)^{2}, \\
& \omega_{-}^{2}=D_{1} D_{2}\left[D_{1}+D_{2}\right]^{-1}(q b)^{2} .
\end{aligned}
$$

Here, $D_{1}=\left[\kappa+\left(2 \kappa^{\prime} / 3\right)\right] \mathrm{m}^{-1}$ and $D_{2}=\left[\kappa^{\prime} / 3\right] \mathrm{M}^{-1}$. Equation (9) is an intermolecular optical mode and acoustic characteristics are seen from (10) in the long-wavelength limit $(q \rightarrow 0)$. The developed modes are alkali- $\mathrm{C}_{60}$ phonon in the $\mathrm{K}_{3} \mathrm{C}_{60}$. We begin with the coupling of conduction electrons with the intermolecular optical vibrations $\left(\omega_{+}\right)$ to seek their role in attractive force for superconductivity.

As an application, the coupling parameters of $\mathrm{K}_{3} \mathrm{C}_{60}$ are evaluated in the following subsection.

\subsection{Electron-phonon attractive parameter}

Superconducting tunneling data yields the electronphonon spectral weight, $\alpha^{2} F(\omega)$ from a strong coupling inversion procedure for the estimation of the coupling constant. Band structure calculation using full potential linear muffin-tin orbital (Novikov et al 1992) method suggests that the superconducting electrons are composed entirely of carbon $2 p$, the contribution of $C 2 s$ orbital is very small and the largest contributions to $N\left(\varepsilon_{\mathrm{F}}\right)$ are provided by the $C(3)$ atoms which are the closest to the neighbouring $\mathrm{C}_{60}$ molecule. It is worth stressing that the 
electron-phonon coupling strength for various phonon modes has been calculated using quantum mechanical (Varma et al 1991) and band structure calculations (Satapthy et al 1992; Erwin and Pickett 1992).

Instead of making use of complicated methods, we use rather simple expressions within McMillan approximation (McMillan 1968) to check whether we find results consistent with the reported data. The use of McMillan expression for the calculation of intermolecular phonon coupling strength is appropriate in $\mathrm{K}_{3} \mathrm{C}_{60}$ superconductors due to the fact that the mass difference of $\mathrm{K}$ and $\mathrm{C}_{60}$ is large. The electron-phonon coupling strength, $\lambda_{\text {er }}$, in this situation is

$$
\lambda_{\text {er }}=\frac{N\left(\varepsilon_{\mathrm{F}}\right)<I^{2}>}{M_{\text {red }}<\omega_{\mathrm{er}}^{2}>},
$$

where $\left\langle I^{2}\right\rangle$ is a mean square electron-ion matrix element, $M_{\text {red }}$ the reduced molecular mass and $\left\langle\omega_{\text {er }}^{2}\right\rangle$ an averaged square molecular vibration frequency. We comment that despite large density of states due to $C(3)$ atoms, (11) may not be used for a crystal with similar mass of carbon in $\mathrm{C}_{60}$. Hence the electron-phonon coupling strength for on-ball- $\mathrm{C}_{60}$ high-energy intramolecular modes needs rigorous quantum mechanical calculations.

The mean square electron-ion matrix element is

$$
\begin{aligned}
<I^{2}>= & \left\{\left[N\left(\varepsilon_{\mathrm{F}}\right)\right]^{2} \int_{0}^{2 k_{\mathrm{F}}}\left(q \mathrm{~d} q / 2 k_{\mathrm{F}}^{2}\right) q^{2}|V(q)|^{2}\right\}\left\{\left[N\left(\varepsilon_{\mathrm{F}}\right)\right]^{2}\right. \\
& \left.\int_{0}^{2 k_{\mathrm{F}}}\left(q \mathrm{~d} q / 2 k_{\mathrm{F}}^{2}\right)\right\}^{-1},
\end{aligned}
$$

$$
=\int_{0}^{2 k_{\mathrm{F}}} q^{3} \mathrm{~d} q|V(q)| / \int_{0}^{2 k_{\mathrm{F}}} q \mathrm{~d} q .
$$

$V(q)$ being the screened Coulomb potential. In terms of bare Coulomb potential, $V_{\mathrm{c}}(q)$, one expresses $V(q)=$ $V_{\mathrm{c}}(q) / \varepsilon(q)$.

Quite generally, the random phase approximation (RPA) properly describes the screening when the kinetic energy is much larger than the interaction energy. Screening in RPA essentially depends on the ratio of effective Coulomb interaction, $U$, and the bandwidth, $W$. In the limit where $U / W(\sim 0.5-1.0)$ i.e. for small values, the RPA underestimates the screening. For large $U / W$, the RPA becomes qualitatively wrong. For intermediate values of $U / W$ $(\sim 1 \cdot 0-2 \cdot 0)$, the RPA yields efficient screening and is the situation in narrow bandwidth alkali intercalated fullerides (Varshney 2000). Looking to the various experimental reports and the fact that for intermediate values of $U / W$, RPA is a valid concept. The idea we have in mind is to deduce the screened Coulomb potential and electronphonon coupling strength within the RPA with a simplified expression on the various physical properties leading to superconducting state.
In particular, within RPA the dielectric function, $\varepsilon(q, \omega)$, for electron with density, $n$, and effective mass, $m^{*}$, in the long-wavelength limit $(q \rightarrow 0)$, is modeled as

$$
\left.\varepsilon(q, \omega)=1+\omega_{\mathrm{p}}^{2}\left[\left(q^{2} v_{\mathrm{F}}^{2} / 2\right)-\omega^{2}\right)\right]^{-1},
$$

with $v_{\mathrm{F}}$ as the Fermi velocity.

The static dielectric function, $\varepsilon(q)$, in the long-wavelength limit is

$$
\varepsilon(q) \approx\left(4 m e^{2} k_{\mathrm{F}}\right)\left[\pi \hbar^{2} q^{2}\right]^{-1},
$$

and the result for screened Coulomb potential is

$$
\begin{aligned}
V(q) & =\frac{4 \pi Z e^{2} /\left(q^{2} \Omega\right)}{4 m e^{2} k_{\mathrm{F}} /\left(\pi \hbar^{2} q^{2}\right)}, \\
& =\left(\hbar^{2} \pi^{2} Z\right)\left[m k_{\mathrm{F}} \Omega\right]^{-1},
\end{aligned}
$$

where $\Omega$ is being the volume of the cell and $Z e$ denotes effective nuclear charge and is $7 \mathrm{e}$.

Thus, the mean square electron ion matrix element from (13) follows

$$
<I^{2}>=\left(\frac{\hbar^{2} \pi^{2} Z}{m k_{\mathrm{F}} \Omega}\right)_{0}^{2 k_{\mathrm{F}}} q^{3} \mathrm{~d} q / 2 k_{\mathrm{F}}^{2},
$$

and the electron-phonon coupling strength for the intermolecular phonon is

$$
\lambda_{\text {er }}=\frac{2 N\left(\varepsilon_{\mathrm{F}}\right)}{M_{\text {red }}<\omega_{\text {er }}^{2}>}\left[\frac{\hbar^{2} \pi^{2} Z}{m \Omega}\right]^{2} .
$$

We may comment on the deduced expression that the electron-phonon coupling strength in fullerides depends very sensitively on the phonon eigen vectors and the electronic structure due to difference between coupling to phonons of different symmetry. We now use the same 3D model to derive the expression for the Coulomb repulsive parameter.

\subsection{Screening parameter}

The effective Coulomb interaction between carriers is introduced by means of the pseudopotential parameter, $\mu^{*}$ (Bogoliubov et al 1959), that we evaluate following the model dielectric function presented in the earlier section. The renormalized Coulomb repulsive parameter is

$$
\mu^{*}=\mu /\left[1+\mu \ln \left\{\varepsilon_{\mathrm{F}} / \omega_{\mathrm{er}}\right\}\right]
$$

We write the Coulomb strength parameter $\mu$ in (20) as $\mu=N\left(\varepsilon_{\mathrm{F}}\right) U$, where $N\left(\varepsilon_{\mathrm{F}}\right)$ is the density of states at the Fermi energy, $\varepsilon_{\mathrm{F}}$ and $U$ the static screened interaction, $V$ $(q, \omega=0)$ averaged over the Fermi sphere. From (15) we have the result

$$
\mu=\frac{1}{2 \pi a_{\mathrm{B}} k_{\mathrm{F}}} \ln \left[1+\frac{3 \pi a_{\mathrm{B}} k_{\mathrm{F}}}{2}\right] .
$$


Having discussed the intermolecular phonon frequencies and coupling strengths, we shall now estimate the superconducting and normal state properties for alkali metal intercalated fullerenes, $\mathrm{K}_{3} \mathrm{C}_{60}$, viz. transition temperature, $T_{\mathrm{c}}$, the carbon isotope effect, $\alpha$, the energy gap ratio $\beta$, the pressure and volume effect on $T_{\mathrm{c}}$, as well as the normal state resistivity and associated transport parameters for doped fullerides.

\section{Discussion and analysis of results}

Any discussion of the fullerides necessitates knowledge of the crystal structure, and this is particularly true of the calculations reviewed here. The $\mathrm{C}_{60}$ molecule is first analysed using the lattice parameter, $a=14.20 \AA$ and $b=18 \mathrm{GPa}$ (Huffman 1991; Hebard 1992). The effective mass of $\mathrm{C}_{60}$ cage is $1.2 \times 10^{-24} \mathrm{~kg}$ and the force constant $\kappa$ is obtained as $19 \cdot 17 \mathrm{~N} / \mathrm{m}$. The estimated value of the longitudinal phonon frequency of the $\mathrm{C}_{60}$ molecule from (5) is $42.42 \mathrm{~cm}^{-1}(5.2 \mathrm{meV})$. The above phonon mode comes from $6 \mathrm{~N}$ bonds between $n-n$ cages of $\mathrm{C}_{60}$ molecule.

Essentially the rotation of $\mathrm{C}_{60}$ molecule is inhibited at temperatures near to room temperature and it appears appropriate to ignore the rotation of $\mathrm{C}_{60}$ molecule in a theory dealing with system at temperatures in the vicinity of $T_{\mathrm{c}}$. Chemical substitution of alkali metal ion in $\mathrm{C}_{60}$ molecule introduces extra electrons which are accommodated in the conduction band which is half filled up to the Fermi level and these conduction electrons distort the lattice of $\mathrm{K}_{3} \mathrm{C}_{60}$. The distortion leads to a net expansion as lattice parameter for $\mathrm{K}$ doped fulleride $(14.28 \AA$ ) and parent fulleride is $a=14 \cdot 20 \AA$. For the evaluation of force constant relationship the total bond energy is then minimized at the experimental value of $a(=14.28 \AA$ ) for $\mathrm{K}_{3} \mathrm{C}_{60}$ to obtain, $\kappa^{\prime}$ as $0 \cdot 17 \kappa$. It appears that the magnitude of force constant for alkali metal intercalated fullerenes is larger than the undoped $\mathrm{C}_{60}$.

The dispersion relations for the intermolecular phonon modes originate due to the displacement of $\mathrm{C}_{60}$ molecule or $\mathrm{K}^{+}$ions in doped fullerides. It is evident from the eigenfrequencies that these correspond to the intermolecular acoustic and alkali- $\mathrm{C}_{60}$ optical modes in the long wavelength limit $(q \rightarrow 0)$. The mass of three alkali metals $(\mathrm{K})$ is used as $0.17 \mathrm{amu}$. The intermolecular alkali $\mathrm{C}_{60}$ optic mode $\left(\omega_{\mathrm{er}}\right)$ is obtained as $7.84 \mathrm{meV}$ at $q \rightarrow 0$. The scattering of charge carriers at the Fermi surface is considered for all possible values of scattering angle, $\theta$. The wave vector, $q\left(\cong 2 k_{\mathrm{F}} \sin \theta\right)$, can, therefore, take maximum value up to $2 k_{\mathrm{F}}$. For small value of $q$, the product $q b$ is 10 to deduce the value of acoustic mode as $125.47 \mathrm{meV}$ at $2 k_{\mathrm{F}}$. In fulleride $\mathrm{K}_{3} \mathrm{C}_{60}$, if all the electrons are free, the electron density, $n\left(=4 \times 3 / a^{3}\right)$, is estimated as $4.12 \times$ $10^{21} \mathrm{~cm}^{-3}$ from the lattice parameter, $a$, of $14.28 \AA$ (Hebard et al 1991). The band structure value of mass, $3.0 m_{\mathrm{e}}$, is used (Novikov et al 1992). Thus the electron parameters as Fermi velocity, Fermi wave vector and plasma fre- quency are obtained as $1.91 \times 10^{7} \mathrm{~cm} \mathrm{~s}^{-1}, 0.496 \AA^{-1}$ and $1.37 \mathrm{eV}$, respectively. Electronic energy band structure calculations (Erwin and Pickett 1992; Satapthy et al 1992) derive the average Fermi velocity as $1.8 \times 10^{7} \mathrm{~cm} \mathrm{~s}^{-1}$ and the plasma frequency of about $1.2 \mathrm{eV}$.

The electron-phonon coupling strength $\left(\lambda_{\text {er }}\right)$ using (19) is obtained as $1 \cdot 2$. We note that the dimensionless electronphonon coupling strength as estimated by Gunnarson is about 0.5-1.0 (Gunnarson 1997). Earlier calculations by Zhang et al (1991a) suggest, however, that the alkali phonons could contribute an attractive interaction of the order of $0.9 \mathrm{eV}$, which corresponds to $\lambda \sim 2.0$ i.e. a strong coupling limit. Later, Burk and coworkers (1994) have shown a null isotope effect with respect to alkali metal dopants, a result which implies that the alkali ions have a weak influence on $T_{\mathrm{c}}$. Theoretical estimates for intermolecular phonon coupling strength is large which is a problem in real sense. It is clear that the enhanced coupling strength is unrealistic. Such strong electron-phonon attraction in fullerides makes the lattice unstable and hence prevents the formation of superconducting state. We argue that the normal state resistivity analysis may provide an interesting check of the value of coupling strength.

The renormalized Coulomb repulsive parameter, $\mu^{*}$, is estimated as $0 \cdot 19$ using (21) and is attributed to the fact that $\varepsilon_{\mathrm{F}}$ is higher atleast an order of magnitude with the intermolecular alkali- $\mathrm{C}_{60}$ optic phonon frequency. Quite generally, the moderately large value of Coulomb repulsive parameter (Gunnarson 1997; Cappelluti et al 2001) is appropriate for a narrow band width material on the metallic side of a metal-insulator transition. To this end, one can see that in dealing with $\mathrm{K}_{3} \mathrm{C}_{60}$ fullerides the coupling strength $(\lambda>1)$ and the renormalized Coulomb repulsive parameter $\left(\mu^{*}=0 \cdot 19\right)$ for the characteristic phonon frequency $\left(\omega_{\mathrm{er}}=7.84 \mathrm{meV}\right)$ distort the lattice leading to a superconducting state.

We now discuss the superconducting transition temperature.

\subsection{Transition temperature}

In the regime, $\lambda>1$, the strong coupling theory applies and we utilize the Kresin $T_{\mathrm{c}}$ expression to get this quantity (Kresin 1987).

$$
T_{\mathrm{c}}^{\mathrm{er}}=0 \cdot 25 \omega_{\mathrm{er}}\left[\exp \left(2 / \lambda_{\mathrm{eff}}\right)-1\right]^{-1 / 2},
$$

where

$$
\lambda_{\text {eff }}=\left(\lambda_{\text {er }}-\mu^{*}\right)\left[1+2 \mu^{*}+\lambda_{\text {er }} \mu^{*} t\left(\lambda_{\text {er }}\right)\right]^{-1},
$$

and

$$
t\left(\lambda_{\mathrm{er}}\right)=1 \cdot 5 \exp \left(-0 \cdot 28 \lambda_{\mathrm{er}}\right) .
$$

The effective coupling constant, $\lambda_{\text {eff }}$, is deduced as 0.62 for the numerical function $t\left(\lambda_{\text {er }}\right)$ value 1.07 with $\lambda_{\text {er }}$ $=1.2$ and $T_{\mathrm{c}}{ }^{\text {er }}$ is estimated as $\cong 5 \mathrm{~K}$ which is much lower 
than the reported experimental data (Hebard et al 1991) of about $18 \mathrm{~K}$. The low value of $T_{\mathrm{c}}$ is due to the fact that the vibrations developed due to the lattice deformations i.e. the soft optical intermolecular phonons, are considered. The above approach with intermolecular phonons does not explain a medium $T_{\mathrm{c}}$ in $\mathrm{K}_{3} \mathrm{C}_{60}$. It is essential to seek the role of high-energy phonons to assess the reported value.

The role of collective excitation mechanism over conventional phonon mechanism in cuprates was earlier successfully explained with a generalized expression for $T_{\mathrm{c}}$ (Kresin 1987). A related problem is that whether one can utilize the broad vibrational spectrum of fullerenes for evaluating $T_{\mathrm{c}}$. With this motivation an analytic result for $T_{\mathrm{c}}$ is employed by incorporating the simultaneous presence of both inter- and intramolecular phonons in fullerides as

$$
T_{\mathrm{c}}=T_{\mathrm{c}}^{\mathrm{er}}\left[\omega_{\mathrm{ra}} / T_{\mathrm{c}}^{\mathrm{er}}\right]^{\chi},
$$

with

$$
\chi=\lambda_{\text {ra }}\left[\lambda_{\text {ra }}+\lambda_{\text {er }}\right]^{-1}
$$

$\lambda_{\text {ra }}$ being the intramolecular coupling constant and is obtained as 0.4 from the scattering time $\left(\tau=1.2 \times 10^{-14} \mathrm{~s}\right)$ (Xiang et al 1993) as

$$
\lambda_{\text {ra }}=\hbar /\left(2 \pi k_{\mathrm{B}} T \tau\right) \text {. }
$$

We use the reported value of high-energy, $H_{\mathrm{g}}$, on-ball$\mathrm{C}_{60}$ molecular phonons, $\omega_{\mathrm{ra}}=1012 \mathrm{~cm}^{-1}$ (Hebard et al 1991; Holczer et al 1991), $T_{\mathrm{c}}$ is obtained as $22 \mathrm{~K}$. It is stressed that $T_{\mathrm{c}}$ is mainly determined by the high-energy, $H_{\mathrm{g}}$, on-ball- $\mathrm{C}_{60}$ molecular phonons. From the analysis of superconducting $T_{\mathrm{c}}$, it is natural to make two comments (i) the high-energy intramolecular phonons are moderately coupled while the low-energy intermolecular phonons are strongly coupled to neighbouring electrons, and (ii) $T_{\mathrm{c}}$ mainly arises from the significant contribution of on-ball- $\mathrm{C}_{60}$ intramolecular phonons.

We now address the screening effects in fullerides. It is known for conventional superconductors, retardation effects in view of different energy scales for electrons and phonons drastically reduce the effects of Coulomb interactions. The dimensionless Coulomb repulsive parameter, $\mu^{*}$, is therefore, severely reduced and attributed to the scaling factor $\ln \left[\varepsilon_{\mathrm{F}} / \omega_{\mathrm{er}}\right]$ appeared in denominator of the expression of $\mu^{*}$. As the Fermi energy is larger atleast by an order of magnitude in intermolecular phonons, the retardation effects are expected to be very small.

On the other hand the Fermi energy and intramolecular phonon frequency are of almost similar order and hence vertex correction becomes important because the Migdal theorem does not hold and the frequency dependence of the effective Coulomb interaction should be considered in fullerides. The above issue will be resolved by considering the Coulomb interactions more carefully. Gene- rally, energy, $\omega_{\mathrm{b}}$, is presented as characterizing bosons $\left(\omega_{\mathrm{er}}\right.$ or $\left.\omega_{\mathrm{ra}}\right)$, but opinion about its choice in screening is far from being unanimous. The only thing one must be sure of is that, if a boson is a candidate for superconductivity, one must expect $\mu^{*}<\mu \sim 0.5$ and of course $\lambda-\mu^{*}$ $>0$. The importance of nonadiabatic effects in fullerides has been discussed earlier at great length (Cappelluti et al 2000, 2001; Alexandrov 2001).

To obtain some specific results, we obtain numerical results (see figure 1) for the transition temperature with intramolecular coupling strength. It is clear that $T_{\mathrm{c}}$ is strongly influenced by the Coulomb repulsive parameter and is higher for small values of $\mu *$. As seen from figure 1 , for higher $\mu^{*}$ value the result is the increased electronelectron repulsive contribution along with the intramolecular phonons producing an attractive interaction is reduced alone against the phonon attraction and attributes to suppressed $T_{\mathrm{c}}$. Higher and positive $\mu^{*}$ implies a constant repulsive interaction that is insufficient to create a superconducting state despite the coupling of intramolecular phonons. Thus Coulomb pseudopotential is an important concept in fullerides. It is noticed from the plot that $T_{\mathrm{c}}$ is highly sensitive to $\lambda_{\mathrm{ra}}$ even for moderate coupling. Thus, if we start with a pure intermolecular phonon mechanism and later on adding a correction term with moderately coupled intramolecular phonons, one can easily enhance $T_{\mathrm{c}}$ values.

An argument that is often put forward with analytical methods is an error bar while estimating transition temperature. In a true sense, the complete calculation, which includes the non-adiabatic effects, carrier mass enhancement effects, exchange and correlations, phase and amplitude fluctuations and narrow band electronic structures of certain materials are quite subtle. Here, in the present study, we argue that the $H_{\mathrm{g}}$ intramolecular phonon besides alkali- $\mathrm{C}_{60}$ phonon is important for electron pairing in fullerides. An often raised query, and one that has caused controversy in the calculations of transition temperature, is: How the vertex corrections and other beyond-

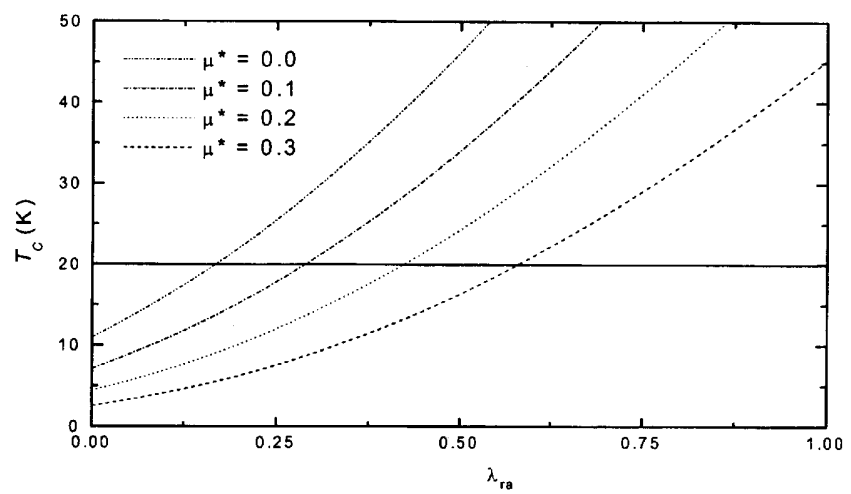

Figure 1. Variation of the superconducting transition temperature with $\lambda_{\rho \alpha}$. Four values of the Coulomb repulsion, $\mu^{*}$, are plotted. Solid line is the experimental value. 
Migdal corrections as well as the momentum dependence of nonadiabatic corrections influence this pairing mechanism, are indeed important issues and will be taken up in near future. However, we admit that the developed mechanism is greatly weakened by these corrections.

Having discussed the transition temperature, we proceed to evaluate the carbon isotope effect coefficient in the next subsection.

\subsection{Isotope effect coefficient}

The isotope effect coefficient is defined as

$$
\alpha=(0 \cdot 5)\left(\mathrm{d} \ln T_{\mathrm{c}} / \mathrm{d} \ln M_{\text {red }}\right) .
$$

The $M_{\text {red }}$ dependence of $T_{\mathrm{c}}$ is introduced in terms of $\mu^{*}$, leading to the following result

$$
\begin{aligned}
\alpha= & \left\{1-\mu^{* 2}\left(1+2 \lambda_{\text {er }}+\lambda_{\text {er }}{ }^{2}\right) / \lambda_{\text {eff }}\left(\lambda_{\text {er }}-\mu^{*}\right)\right. \\
& {\left.\left[1+\left(4 T_{\mathrm{c}} / \omega\right)^{2}\right]\right\} / 2, }
\end{aligned}
$$

with $\omega$ as the average phonon frequency.

Equation (29) yields $\alpha \cong 0.24$ which is slightly lower than the reported value of $\alpha=0.30 \pm 0.06$ with $100 \%$ substitution of ${ }^{6} C_{13}$ for ${ }^{6} C_{12}$ (Chen and Lieber 1993) and consistent with results of Fuhrer et al (1999). Figure 2 shows the variation of estimated isotope effect from (29) with screening parameter. It is noticed that the BCS one half value is recovered for $\mu^{*}=0 \cdot 0$. With the increase in $\mu^{*}$ values the isotope effect exponent decreases for $\lambda_{\text {er }}=1.2$ and $\lambda_{\text {ra }}=0.4$.

The intermolecular phonon frequency is dominated by the mass of alkali metal as $\omega_{\mathrm{er}} \sim[m M /(m+M)]^{-1 / 2}$ with $m$ $(M)$ mass of $\mathrm{C}_{60}(\mathrm{~K})$ yielding a nearly zero $\alpha$ value. However, the mass of $\mathrm{C}_{60}$ makes a noticeable change in the intramolecular phonon frequency, as $\omega_{\mathrm{ra}} \sim[(m+M) / 2]^{-1 / 2}$, leading to a finite value of carbon isotope effect. Thus alkali metal isotope essentially changes intermolecular vibrations as the reduced interfullerene mass $[\mathrm{mM} /(m+M)]$

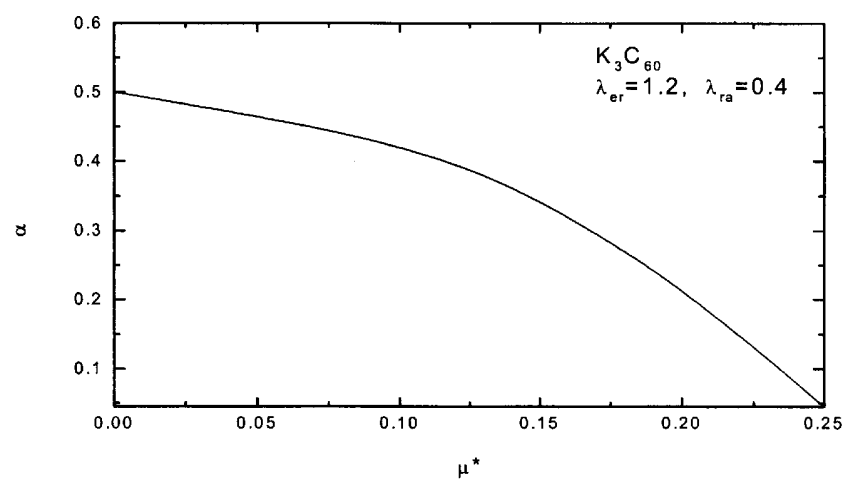

Figure 2. Variation of isotope effect exponent with Coulomb pseudopotential. is small in comparison with the intrafullerene mass $[(m+M) / 2]$. Furthermore, the null alkali metal isotope points to the fact that the interfullerene isotopic disorder reduces the $T_{\mathrm{c}}$ more than does the intrafullerene isotopic disorder. Thus, the alkali metal ion modes contribute very little to superconducting state of fullerides and intramolecular phonons, which are moderately coupled, conceive the superconducting state in fullerides.

What is required is the total isotope effect to assess the role of electron-phonon in the pairing mechanism. However, a reliable alkali metal isotope effect is not reported for $\mathrm{K}_{3} \mathrm{C}_{60}$ system, but a negative $\alpha_{\mathrm{Rb}}$ is documented (Burk et al 1994). The requirement is to know either the ratio $\alpha_{\mathrm{c}} / \alpha_{\mathrm{K}}$ or the total isotope effect to have a final conclusion about the role of inter- or intramolecular phonons in the superconducting state based on isotope effect exponent. The justification lies in a fact that the ratio $\alpha_{c} / \alpha_{K}$ depends on the weights of on ball carbon and $\mathrm{C}_{60}$-alkali metal dominated phonon modes that are sensitive to coupling strength parameters. We argue that the Coulomb interactions, multiatomic compounds, anharmonicity, and nonphononic mechanisms may affect the value of isotope effect exponent and needs detailed investigations. As a next step we focus on the magnitude of the energy gap parameter.

\subsection{Energy gap parameter}

The energy gap parameter, $\beta$ (Kresin 1987), is

$$
\beta \cong 2 \Delta(0) / k_{\mathrm{B}} T_{\mathrm{c}}=3 \cdot 25\left[1+5\left(T_{\mathrm{c}} / \omega_{\mathrm{ra}}\right)^{2} \ln \left(\omega_{\mathrm{ra}} / T_{\mathrm{c}}\right)\right],
$$

where $\Delta(0)$ is the energy gap at zero temperature. The gap parameter when intramolecular phonons are alone considered in the pairing mechanism is estimated as 3.54 which is close to the BCS limit where the values, $\omega_{\mathrm{ra}}=$ $1012 \mathrm{~cm}^{-1}$ and $T_{\mathrm{c}}=22 \mathrm{~K}$, are used. This implies that, the intramolecular phonons require moderate interactions with the conduction electrons and is consistent with the infrared spectroscopy (DeGiorgi et al 1994), muon spin relaxation rate measurements (Kiefl et al 1993) and nuclear spin relaxation measurements (Tycko et al 1992), which strongly favour the participation of intramolecular phonons in the pairing mechanism. Furthermore, the absolute reflectivity measurements (DeGiorgi et al 1992) on $\mathrm{K}_{3} \mathrm{C}_{60}$ reports $\beta=3.6 \sim 4.0$, which is similar to $\mathrm{BCS}$ weak coupling limit and the electron-phonon coupling strength cannot be as large as 2 .

The Fermi velocity, $v_{\mathrm{F}}$, along with a $T_{\mathrm{c}}$ of $22 \mathrm{~K}$ leads to a BCS coherence length of $12.9 \mathrm{~nm}$ using the precise definition

$$
\xi_{0} \cong \hbar v_{\mathrm{F}} / 1 \cdot 76 \pi k_{\mathrm{B}} T_{\mathrm{c}},
$$

which is consistent with the value of 12.0 (Ramirez et al 1992), 13.0 $\pm 0 \cdot 15$ (Hou et al 1993) and $15 \cdot 0 \mathrm{~nm}$ (Palstra et al 1992), for $\mathrm{K}_{3} \mathrm{C}_{60}$ fullerenes. It is interesting to com- 
ment that the coherence length which, even if small, is much larger than inverse Fermi momentum. We further estimate the zero temperature mean free path, $\ell=v_{\mathrm{F}} \tau$ $\approx 3.4 \mathrm{~nm}$. The small value of $\ell$ is mainly attributed to the disorder, which is present in doped fulleride samples, including the best available single crystals. The effective coherence length following $\xi_{00}{ }^{-1}=\xi_{0}{ }^{-1}+\ell^{-1}$ leads to a value of about $2.7 \mathrm{~nm}$ consistent with the earlier estimates of 2.6-4.5 nm (Sparn et al 1991, 1992). Hence, the alkali metal doped fullerides are dirty superconductors defined by $\ell \leq \xi_{0}, \xi_{00}$.

The magnetic penetration depth at $T=0 \mathrm{~K}$ as $\lambda_{\mathrm{L} 0}$ $\left[=\sqrt{ }\left(m^{*} c^{2} / 4 \pi n \mathrm{e}^{2}\right)\right]$ is estimated as $143.6 \mathrm{~nm}$ which is slightly smaller than the values of $156 \mathrm{~nm}$ (Hou et al 1995 ) in $\mathrm{K}_{3} \mathrm{C}_{60}$. The consistency is attributed to the proper choice of transport parameters as effective mass of carriers and carrier density. The effective penetration depth, $\left\{\lambda_{\mathrm{L} 00}=\lambda_{\mathrm{L} 0} \sqrt{ }\left[1+\xi_{00} \ell^{-1}\right]\right\}$, is about $192 \mathrm{~nm}$ consistent with the earlier estimate of $240 \pm 30 \mathrm{~nm}$ (Hou et al 1995), $600 \mathrm{~nm}$ (Tycko et al 1992) and $800 \mathrm{~nm}$ (DeGiorgi et al 1992). Given the numbers that emerge from the analysis, it seems fair to conclude that fullerides belong to conventional $s$ wave superconductor. We now discuss the method of calculation for pressure and volume effect on superconducting transition temperature in the test material.

\subsection{Pressure effect on transition temperature}

To analyse the pressure dependence of $T_{\mathrm{c}}$ for $\mathrm{K}_{3} \mathrm{C}_{60}$ system, we begin with (25) according to which,

$$
\begin{aligned}
\frac{\mathrm{d} \ln T_{\mathrm{c}}}{\mathrm{d} P}= & {\left[1-\frac{\lambda_{\mathrm{ra}}}{\lambda_{\mathrm{er}}+\lambda_{\mathrm{ra}}}\right] \frac{\mathrm{d} \ln T_{\mathrm{c}}^{\mathrm{er}}}{\mathrm{d} P}+} \\
& {\left[\ln \omega_{\mathrm{ra}}-\ln T_{\mathrm{c}}^{\mathrm{er}}\right] \frac{\mathrm{d}}{\mathrm{d} P}\left[\frac{\lambda_{\mathrm{ra}}}{\lambda_{\mathrm{er}}+\lambda_{\mathrm{ra}}}\right]+} \\
& {\left[\frac{\lambda_{\mathrm{ra}}}{\lambda_{\mathrm{er}}+\lambda_{\mathrm{ra}}}\right] \frac{\mathrm{d} \ln \omega_{\mathrm{ra}}}{\mathrm{d} P} . }
\end{aligned}
$$

Keeping in mind that $\mathrm{C}_{60}$ molecules are extremely rigid and hence neglecting the pressure induced effects of intramolecular phonons,

$$
\frac{\mathrm{d} \ln \omega_{\mathrm{ra}}}{\mathrm{d} P}=\frac{\mathrm{d} \omega_{\mathrm{ra}}}{\mathrm{d} P}=0
$$

Henceforth, we rewrite (31) as

$$
\begin{aligned}
\frac{\mathrm{d} \ln T_{\mathrm{c}}}{\mathrm{d} P}= & {\left[1-\frac{\lambda_{\mathrm{ra}}}{\lambda_{\mathrm{ra}}+\lambda_{\mathrm{er}}}\right] \frac{\mathrm{d} \ln T_{\mathrm{c}}^{\mathrm{er}}}{\mathrm{d} P}+\frac{1}{\left(\lambda_{\mathrm{er}}+\lambda_{\mathrm{ra}}\right)^{2}} } \\
& {\left[\ln \left(\frac{\omega_{\mathrm{ra}}}{T_{\mathrm{c}}^{\mathrm{er}}}\right)\right]\left[\lambda_{\mathrm{er}} \frac{\lambda_{\mathrm{ra}}}{\mathrm{d} P}-\lambda_{\mathrm{ra}} \frac{\mathrm{d} \lambda_{\mathrm{er}}}{\mathrm{d} P}\right] . }
\end{aligned}
$$

Furthermore,

$$
\frac{\mathrm{d} \ln T_{\mathrm{c}}^{\mathrm{er}}}{\mathrm{d} P}=\frac{\partial \ln T_{\mathrm{c}}^{\mathrm{er}}}{\partial \lambda_{\mathrm{er}}} \frac{\mathrm{d} \lambda_{\mathrm{er}}}{\mathrm{d} P}+\frac{\partial \ln T_{\mathrm{c}}^{\mathrm{er}}}{\partial \omega_{\mathrm{er}}} \frac{\mathrm{d} \omega_{\mathrm{er}}}{\mathrm{d} P}+\frac{\partial \ln T_{\mathrm{c}}^{\mathrm{er}}}{\partial \mu^{*}} \frac{\mathrm{d} \mu^{*}}{\mathrm{~d} P} .
$$

Using (22), we get the following results

$$
\frac{\partial \ln T_{\mathrm{c}}^{\mathrm{er}}}{\partial \lambda_{\mathrm{er}}} \frac{\mathrm{d} \lambda_{\mathrm{er}}}{\mathrm{d} P}=D_{11} \frac{\mathrm{d} \ln \lambda_{\mathrm{er}}}{\mathrm{d} P}
$$

where

$$
\begin{aligned}
D_{11}= & \frac{\lambda_{\text {er }}\left(1+2 \mu^{*}+1 \cdot 07 \mu^{*^{2}}\right)}{\left(\lambda_{\text {er }}-\mu^{*}\right)^{2}} \\
& {\left[\begin{array}{l}
1+\left\{\exp \left(\frac{2+4 \mu^{*}+2 \cdot 14 \lambda_{\text {er }} \mu^{*}}{\lambda_{\text {er }}-\mu^{*}}\right)\right\}^{-1} \\
+\left\{\exp \left(\frac{2+4 \mu^{*}+2 \cdot 14 \lambda_{\text {er }} \mu^{*}}{\lambda_{\text {er }}-\mu^{*}}\right)\right\}^{-2}
\end{array}\right] }
\end{aligned}
$$

We use the following

$$
\frac{\partial \ln T_{\mathrm{c}}^{\mathrm{er}}}{\partial \omega_{\mathrm{er}}} \frac{\mathrm{d} \omega_{\mathrm{er}}}{\mathrm{d} P}=\frac{\mathrm{d} \ln \omega_{\mathrm{er}}}{\mathrm{d} P},
$$

and

$$
\frac{\partial \ln T_{\mathrm{c}}^{\mathrm{er}}}{\partial \mu^{*}} \frac{\mathrm{d} \mu^{*}}{\mathrm{~d} P}=D_{12} \frac{\mathrm{d} \ln \mu^{*}}{\mathrm{~d} P},
$$

where

$$
\begin{aligned}
D_{12}= & -\frac{\mu *\left(1+2 \lambda_{\mathrm{er}}+1 \cdot 07 \lambda_{\mathrm{er}}^{2}\right)}{\left(\lambda-\mu^{*}\right)^{2}} \\
& {\left[\begin{array}{l}
1+\left\{\exp \left(\frac{2+4 \mu^{*}+2 \cdot 14 \lambda_{\mathrm{er}} \mu^{*}}{\lambda_{\mathrm{er}}-\mu^{*}}\right)\right\}^{-1} \\
+\left\{\exp \left(\frac{2+4 \mu^{*}+2 \cdot 14 \lambda_{\mathrm{er}} \mu^{*}}{\lambda_{\mathrm{er}}-\mu^{*}}\right)\right\}^{-2}
\end{array}\right] . }
\end{aligned}
$$

Combining these, we write

$$
\frac{\mathrm{d} \ln T_{\mathrm{c}}^{\mathrm{er}}}{\mathrm{d} P}=D_{11} \frac{\mathrm{d} \ln \lambda_{\mathrm{er}}}{\mathrm{d} P}+\frac{\mathrm{d} \ln \omega_{\mathrm{er}}}{\mathrm{d} P}+D_{12} \frac{\mathrm{d} \ln \mu^{*}}{\mathrm{~d} P} .
$$

The first and the third terms are dependent on the variation of the $N\left(\varepsilon_{\mathrm{F}}\right)$ with pressure.

We proceed for further calculation by using the reported value of $B, 13.18 \mathrm{GPa}$ (Huffman 1991) and calculated $\varepsilon_{\mathrm{F}}, 0.31 \mathrm{eV}$, as documented in the previous section. The free electron gas result $\operatorname{d} \ln N\left(\varepsilon_{\mathrm{F}}\right) / \mathrm{d} P=(-2 / 3) \kappa_{\mathrm{T}}$, 
where $\kappa_{\mathrm{T}}\left(\sim B^{-1}\right)$ is thermal compressibility. Deduced value of $\mathrm{d} \ln N\left(\varepsilon_{\mathrm{F}}\right) / \mathrm{d} P$ is about $-5.058 \%(\mathrm{GPa})^{-1}$. Kerkoud and coworkers (1994) reported a rate of about $\sim-10 \%$ $(\mathrm{GPa})^{-1}$ from NMR technique although of limited accuracy.

From (11) and (13), we write

$$
\begin{aligned}
\frac{\mathrm{d} \ln \mu^{*}}{\mathrm{~d} P}= & \frac{\mathrm{d} \ln N\left(\varepsilon_{\mathrm{F}}\right)}{\mathrm{d} P}\left(\frac{\mu^{*}}{\mu}\right)-\mu^{*} \\
& {\left[\frac{2 \mathrm{~d} \ln \omega_{p}}{\mathrm{~d} P}-\frac{\mathrm{d} \ln N\left(\varepsilon_{\mathrm{F}}\right)}{\mathrm{d} P}-\frac{\mathrm{d} \ln \omega_{\mathrm{er}}}{\mathrm{d} P}\right], } \\
\frac{\mathrm{d} \ln \lambda_{\mathrm{er}}}{\mathrm{d} P}= & \frac{\mathrm{d} \ln N\left(\varepsilon_{\mathrm{F}}\right)}{\mathrm{d} P}-\frac{\mathrm{d} \ln \left\langle\omega_{\mathrm{er}}^{2}\right\rangle}{\mathrm{d} P} .
\end{aligned}
$$

Herein, the pressure dependence of the quantity $\left\langle I^{2}\right\rangle$ has been neglected, as the mean-square electron-ion matrix element is independent of phonon frequencies. Assuming that delta functions entering the integrals in (12) does not vary much under pressure, we neglect the pressure dependence of the quantity $\left\langle I^{2}\right\rangle$. Stated in another way the physical quantities appearing in (11) are unaffected under pressure.

Using (41) and (42) into (40), we obtain

$$
\begin{aligned}
\frac{\mathrm{d} \ln T_{\mathrm{c}}^{\mathrm{er}}}{\mathrm{d} P} & =\left[D_{11}+D_{12}\left(\frac{\mu^{*}}{\mu}\right)+D_{12} \mu^{*}\right] \frac{\mathrm{d} \ln N\left(\varepsilon_{\mathrm{F}}\right)}{\mathrm{d} P} \\
& +\left[1+D_{12} \mu^{*}-2 D_{11}\right] \frac{\mathrm{d} \ln \left\langle\omega_{\mathrm{er}}\right\rangle}{\mathrm{d} P}-2 D_{12} \mu^{*} \frac{\mathrm{d} \ln \omega_{\mathrm{p}}}{\mathrm{d} P} .
\end{aligned}
$$

In order to make progress we assume $\mathrm{d} \ln \lambda_{\text {ra }} / \mathrm{d} P \approx \mathrm{d} \ln N$ $\left.\left(\varepsilon_{\mathrm{F}}\right) / \mathrm{d} P, \mathrm{~d} l n<\omega_{\mathrm{er}}^{2}\right\rangle / \mathrm{d} P \approx 2 \mathrm{dln}\left\langle\omega_{\mathrm{er}}\right\rangle / \mathrm{d} P$ and $\mathrm{dln}\left\langle\omega_{\mathrm{er}}\right\rangle / \mathrm{d} P \approx$ $\mathrm{d} \ln \left\langle\omega_{\mathrm{er}}\right\rangle / \mathrm{d} P$. The free electron estimate for $\mathrm{d} \ln \omega_{\mathrm{p}} / \mathrm{d} P=$ $1 / 2 B$ yields a value of about $3.79 \%(\mathrm{GPa})^{-1}$. We follow $\gamma_{\mathrm{G}}=B$ dln $\langle\omega\rangle / \mathrm{d} P$ to obtain $\operatorname{dln} \omega_{\mathrm{er}} / \mathrm{d} P=15 \cdot 17 \%(\mathrm{GPa})^{-1}$, using $\gamma_{\mathrm{G}}=+2$ as reported earlier (Diederich et al 1996). The parameters needed for further calculations are obtained as $D_{11}=1.7$ and $D_{12}=-0.96$ from the values of $\lambda=1 \cdot 2, \mu=0.75$ and $\mu^{*}=0 \cdot 19$. Using (33)-(43) we find the value of $\mathrm{d} \ln \mu^{*} / \mathrm{d} P=-0.78 \%(\mathrm{GPa})^{-1}, \mathrm{~d} \ln \lambda_{\mathrm{er}} / \mathrm{d} P=$ $-35.39 \%(\mathrm{GPa})^{-1}, \mathrm{~d} \ln \lambda_{\mathrm{ra}} / \mathrm{d} P=-5.05 \%(\mathrm{GPa})^{-1}, \mathrm{~d} \ln T_{\mathrm{c}}^{\mathrm{er}} / \mathrm{d} P$ is $-45.64 \%(\mathrm{GPa})^{-1}, \mathrm{~d} \ln T_{\mathrm{c}} / \mathrm{d} P=-39.60 \%(\mathrm{GPa})^{-1}$ and slope $\mathrm{d} T_{\mathrm{c}} / \mathrm{d} P=-8.7 \mathrm{~K} / \mathrm{GPa}$ which is consistent with the earlier reported value (Sparn et al 1991, 1992) of $-7 \cdot 8 \mathrm{~K} / \mathrm{GPa}$.

Figure 3 shows the variation of $T_{\mathrm{c}}$ with pressure along with the experimental data. For fitting of the data we have employed the relation, $T_{\mathrm{c}}(P)=T_{\mathrm{c}}(0) \exp [-0 \cdot 3 \gamma P]$, using $\gamma_{\mathrm{G}}=+2$ (Diederich et al 1996). Here, the logarithmic derivative of the Coulomb pseudopotential with pressure, $\mathrm{d} \ln \mu^{*} / \mathrm{d} P$, is smaller as compared to the other logarithmic derivatives involved in the expression of superconducting transition temperature.

In principle, the electron-intermolecular phonon coupling parameter is directly proportional to the density of states at the Fermi level and inversely proportional to appropriately averaged square of the intermolecular optical phonon frequency. The variation of $T_{\mathrm{c}}$ with pressure is thus dependent on the variation of the density of states at Fermi level, the Coulomb pseudopotential and the variation of the characteristic phonon frequency with pressure. Due to extreme rigidity of $\mathrm{C}_{60}$, the pressure dependence of on-ball- $\mathrm{C}_{60}$ phonon frequencies are neglected. To test this idea, it will be of interest to investigate the volume derivative of $T_{\mathrm{c}}$.

\subsection{Volume dependent transition temperature}

We now explore our efforts in studying the volume derivative of $T_{\mathrm{c}}$ or $\mathrm{d} T_{\mathrm{c}} / \mathrm{d} V$. We note that due to extreme rigidity of $\mathrm{C}_{60}$ the compression contracts the weak intermolecular bonds and high-energy intramolecular bonds are unaffected. The volume derivative of $T_{\mathrm{c}}$ from (25) reads,

$$
\frac{\mathrm{d} T_{\mathrm{c}}}{\mathrm{d} V}=T_{\mathrm{c}}^{\mathrm{er}} \cdot \frac{\mathrm{d}}{\mathrm{d} V}\left[\frac{\omega_{\mathrm{ra}}}{T_{\mathrm{c}}^{\mathrm{er}}}\right]^{\chi}+\left[\frac{\omega_{\mathrm{ra}}}{T_{\mathrm{c}}^{\mathrm{er}}}\right]^{\chi} \cdot \frac{\mathrm{d} T_{\mathrm{c}}^{\mathrm{er}}}{\mathrm{d} V} .
$$

We further write

$$
\frac{\mathrm{d} T_{\mathrm{c}}}{\mathrm{d} V}=(1-\chi)\left[\frac{\omega_{\mathrm{ra}}}{T_{\mathrm{c}}^{\mathrm{er}}}\right]^{\chi} \frac{\mathrm{d} T_{\mathrm{c}}^{\mathrm{er}}}{\mathrm{d} V} .
$$

Herein, it is assumed that intramolecular phonons are independent in any change of the volume (Crespi and Cohen 1996),

$$
\frac{\mathrm{d} \ln \omega_{\mathrm{ra}}}{\mathrm{d} V}=\frac{\mathrm{d} \omega_{\mathrm{ra}}}{\mathrm{d} V}=0 .
$$

Following (22), we write

$$
\begin{aligned}
\frac{\mathrm{d} T_{\mathrm{c}}^{\mathrm{er}}}{\mathrm{d} V}= & \frac{0 \cdot 25}{\left[\exp \left(2 / \lambda_{\text {eff }}\right)-1\right]^{1 / 2}} \times \frac{\mathrm{d} \omega_{\text {er }}}{\mathrm{d} V} \\
& +\frac{\exp \left(2 / \lambda_{\text {eff }}\right)}{\left[\exp \left(2 / \lambda_{\text {eff }}\right)-1\right]^{3 / 2}} \times\left(\frac{0 \cdot 25 \omega_{\text {er }}}{\lambda_{\text {eff }}^{2}}\right) \frac{\mathrm{d} \lambda_{\text {eff }}}{\mathrm{d} V},
\end{aligned}
$$

where,

$$
\begin{aligned}
\frac{d \lambda_{\text {eff }}}{d V}= & \left(1+2 \mu^{*}+\mu^{* 2} t\left(\lambda_{\text {er }}\right)\right) \frac{\mathrm{d} \lambda_{\text {er }}}{d V}+ \\
& \left(\mu^{*} \lambda_{\text {er }}-\mu^{*} \lambda_{\text {er }}^{2}\right) \frac{d t\left(\lambda_{\text {er }}\right)}{d V}- \\
& \left(1+2 \lambda_{\text {er }}+2 \lambda_{\text {er }}^{2} t\left(\lambda_{\text {er }}\right)\right) \frac{\mathrm{d} \mu^{*}}{\mathrm{~d} V} .
\end{aligned}
$$

Using (49) and (47), we write

$$
\frac{\mathrm{d} T_{\mathrm{c}}}{\mathrm{d} V}=D_{21} \frac{\mathrm{d} \omega_{\mathrm{er}}}{\mathrm{d} V}+D_{22} \frac{\mathrm{d} \lambda_{\mathrm{er}}}{\mathrm{d} V}+D_{23} \frac{\mathrm{d} t\left(\lambda_{\mathrm{er}}\right)}{\mathrm{d} V}-D_{24} \frac{\mathrm{d} \mu^{*}}{\mathrm{~d} V}
$$


Here, we define

$$
\begin{aligned}
D_{21}= & V_{00}\left\{\frac{0 \cdot 25}{\left(\exp \left(2 / \lambda_{\text {eff }}\right)-1\right)^{1 / 2}}\right\}, \\
D_{22}= & V_{00}\left\{\frac{\exp \left(2 / \lambda_{\text {eff }}\right)}{\left(\exp \left(2 / \lambda_{\text {eff }}\right)-1\right)^{3 / 2}} \times \frac{0 \cdot 25 \omega_{\text {er }}}{\lambda_{\text {eff }}^{2}}\right\} \\
& \left(1+2 \mu^{*}+\mu^{* 2} t\left(\lambda_{\text {er }}\right)\right) \\
D_{23}= & V_{00}\left\{\frac{\exp \left(2 / \lambda_{\text {eff }}\right)}{\left(\exp \left(2 / \lambda_{\text {eff }}\right)-1\right)^{3 / 2}} \times \frac{0 \cdot 25 \omega_{\text {er }}}{\lambda_{\text {eff }}^{2}}\right\} \\
& \left(\mu^{*} \lambda_{\text {er }}-\mu^{*} \lambda_{\text {er }}{ }^{2}\right),
\end{aligned}
$$

and

$$
\begin{aligned}
D_{24}= & -V_{00}\left\{\frac{\exp \left(2 / \lambda_{\text {eff }}\right)}{\left(\exp \left(2 / \lambda_{\text {eff }}\right)-1\right)^{3 / 2}} \times \frac{0 \cdot 25 \omega_{\text {er }}}{\lambda_{\text {eff }}^{2}}\right\} \\
& \left(1+2 \lambda_{\text {er }}+2 \lambda_{\text {er }}^{2} t(\lambda)_{\text {er }}\right),
\end{aligned}
$$

where

$$
V_{00}=\left(\omega_{\mathrm{ra}} / T_{\mathrm{c}}^{\mathrm{er}}\right)^{\frac{\lambda_{\mathrm{ra}}}{\lambda_{\mathrm{er}}+\lambda_{\mathrm{ra}}}}\left(1-\frac{\lambda_{\mathrm{ra}}}{\lambda_{\mathrm{er}}+\lambda_{\mathrm{ra}}}\right) .
$$

Furthermore, the logarithmic volume derivative of the coupling parameter $\lambda_{\text {er }}$ follows:

$$
\Phi=\frac{\mathrm{d} \ln \lambda_{\mathrm{er}}}{\mathrm{d} \ln V}=B \frac{\mathrm{d} \ln N\left(\varepsilon_{\mathrm{F}}\right)}{\mathrm{d} P}+2 \gamma_{\mathrm{G}},
$$

with the values of $B, \operatorname{d} \ln N\left(\varepsilon_{\mathrm{F}}\right) / \mathrm{d} P$ and $d \ln \langle\omega\rangle / \mathrm{d} P$ discussed earlier, we find $\Phi=3.3$ for $\mathrm{K}_{3} \mathrm{C}_{60}$. To ascertain the physical significance of the volume derivative of $T_{\mathrm{c}}$ we estimate the parameters as $D_{21}=0.16, D_{22}=70.6$, $D_{23}=-2.7$ and $D_{24}=-323$ from the earlier mentioned

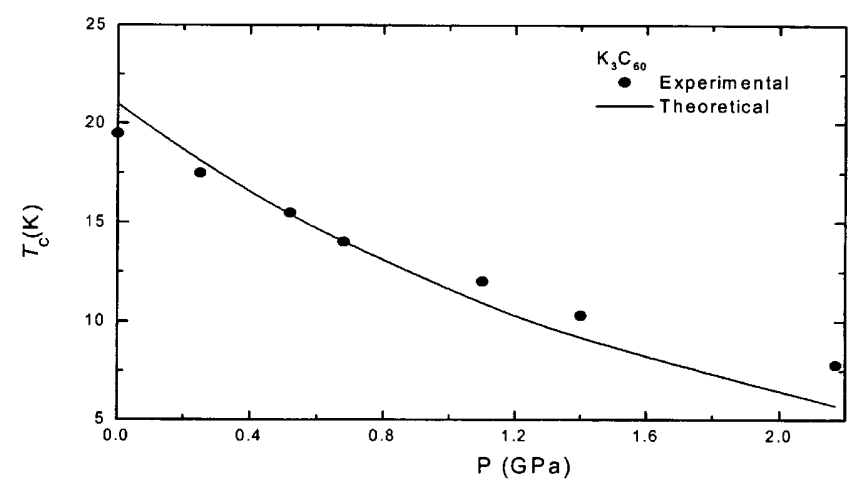

Figure 3. The dependence of $T_{\mathrm{c}}$ with pressure. Experimental data (closed circles) taken from Sparn et al $(1991,1992)$. values of various coupling strengths. Looking to (49), $\mathrm{d} T_{\mathrm{c}} / \mathrm{d} V$ is being influenced by (a) volume dependence of screening parameter and (b) volume dependence of intermolecular vibrational mode as well as intramolecular phonon coupling strength. Because of the difficulties in determining the volume derivative of molecular phonon frequency, we are only able to provide some suggestive formal argument below.

Usually, the compression increases the bandwidth and hence $\varepsilon_{\mathrm{F}}$ resulted in the reduction of renormalized screening parameter. We note that the Coulomb repulsion suppresses $T_{\mathrm{c}}$ as is being noticed from figure 1. Henceforth, the contribution to $\mathrm{d} T_{\mathrm{c}} / \mathrm{d} V$ proportional to $\mathrm{d} \mu{ }^{*} / \mathrm{d} V$ is negative. The logarithmic dependence of $\mu^{*}$ on $\varepsilon_{\mathrm{F}}$ points to the fact that volume derivative of $\mu^{*}$ is small in magnitude. A natural argument follows that the volume derivative of $t\left(\lambda_{\text {er }}\right)$ and of $\mu^{*}$ is small in comparison to $\mathrm{d} \lambda_{\mathrm{er}} / \mathrm{d} V$, henceforth we neglect these two terms in (49). In summary, intermolecular phonon frequencies are influenced under compression modes and if superconductivity in the fullerenes arose from coupling to intermolecular phonons then this argument implies strong electron-phonon coupling. We write the logarithmic volume derivative of $T_{\mathrm{c}}$ in terms of bulk modulus and its lattice parameter dependence as

$$
\frac{\mathrm{d} \ln T_{\mathrm{c}}}{\mathrm{d} \ln V}=-\frac{\mathrm{d} \ln T_{\mathrm{c}}}{\mathrm{d} P} B=\frac{\mathrm{d} \ln T_{\mathrm{c}}}{3 \mathrm{~d} \ln a} .
$$

As $\mathrm{d} T_{\mathrm{c}} / \mathrm{d} P$ is negative, we find the volume derivative of $T_{\mathrm{c}}$ is positive and large in fullerides.

We now discuss and compute numerically the response of resistivity in $\mathrm{K}_{3} \mathrm{C}_{60}$.

\subsection{Zero temperature limited resistivity}

In usual metals the electron-phonon scattering is a major source of temperature dependent resistivity and can describe the normal state transport properties. However, apart from electron-phonon scattering, other scattering mechanisms as electrons scatter off impurities, defects, grain boundaries and disordered regions, leads to a temperature independent contribution. We begin with the latter contribution to resistivity.

Information of zero temperature elastic scattering rate and plasma frequency will allow us to have an independent estimation of zero temperature-limited resistivity. The zero temperature scattering rates is related through the upper critical magnetic field, $H_{\mathrm{c} 2}(0)$. Following the two-squarewell analysis of Eliashberg theory, Carbotte (1990) suggested that the strong coupling corrections are important and a rescaling factor of $1+\lambda$ appears in the modified BCS results. The Matsubara gap function, which is related with upper critical magnetic field yields

$$
\frac{1+\lambda}{\lambda-\mu^{*}}=2 \pi \frac{T}{T_{\mathrm{c}}} \sum_{m=0}^{N_{\mathrm{c}}} \frac{1}{\chi_{\mathrm{m}}^{-1}\left(\bar{\omega}_{\mathrm{m}}\right)-(2 \tau)^{-1}} .
$$


$\bar{\omega}_{\mathrm{m}}$ being the Matsubara frequency, within the standard two-square-well model and is $\bar{\omega}_{\mathrm{m}}=\omega_{\mathrm{m}}(1+\lambda)+(2 \tau)^{-1}$ $\left(\operatorname{sgn} \omega_{\mathrm{m}}\right), \lambda$ the electron-phonon coupling strength with cut off at $N_{\mathrm{c}}$ and $\tau$ the scattering time. In this approximation, $N_{\mathrm{c}}$ follows

$$
N_{\mathrm{c}}=(1 / 2)[(\omega / \pi T)+1] \text {. }
$$

$\mu^{*}$ being the renormalized Coulomb repulsive parameter and the factor $\chi_{\mathrm{m}}$ appearing in (57) is

$$
\chi_{\mathrm{m}}\left(\bar{\omega}_{\mathrm{m}}\right)=\frac{2}{\sqrt{\xi^{*}}} \int_{0}^{\infty} \exp \left(-q^{2}\right) \tan ^{-1}(\phi) \mathrm{d} q
$$

with

$$
\phi=\frac{q \sqrt{\xi^{*}}}{\left[(2 m+1) \pi \frac{T}{T_{\mathrm{c}}}\right]+\left[\frac{1}{2 \tau^{*}}\right]} .
$$

The upper critical magnetic field is related through

$$
\xi^{*}=\frac{1}{2} e H_{\mathrm{c} 2}^{*} v_{\mathrm{F}}^{* 2} \text {. }
$$

The physical quantities appearing in (57)-(61) involve renormalized values as

$$
\begin{aligned}
& H_{\mathrm{c} 2}^{*}=\frac{H_{\mathrm{c} 2}}{(1+\lambda) T_{\mathrm{c}}}, \\
& v_{\mathrm{F}}^{*}=\frac{v_{\mathrm{F}}}{\sqrt{(1+\lambda) T_{\mathrm{c}}}},
\end{aligned}
$$

and impurity scattering time

$$
\tau^{*}=\frac{\tau}{(1+\lambda) T_{\mathrm{c}}}
$$

The above derived equations differ from the BCS limit, as the renormalizations in $\xi^{*}, v_{\mathrm{F}}{ }^{*}, H^{*}{ }_{\mathrm{c} 2}$ and $\tau^{*}$ are introduced. These expressions are valid for any impurity concentration described in (57)-(61) by scattering time. In the present analysis, Pauli limit has been neglected as an approximation (Grimvall 1981), due to relatively small value of $\mathrm{d} H_{\mathrm{c} 2} / \mathrm{d} T[1 /(1+\lambda)]$ in alkali metal intercalated fullerenes. In principle, the above approach describes quantitatively the renormalization of the physical properties due to electron-phonon interaction and is therefore reduced by $1+\lambda$.

The zero temperature-limited resistivity is expressed as

$$
\rho(0)=4 \pi \tau^{*-1} \omega_{\mathrm{p}}^{-2} \text {. }
$$

From the above it is noticed that the determination of scattering rate essentially needs the Coulomb repulsive parameter, electron-phonon coupling strength, Fermi velocity, plasma frequency and upper critical magnetic field. This allows one to estimate the zero temperature limited resistivity independently. We use the earlier deduced value of $T_{\mathrm{c}}=22 \mathrm{~K}, v_{\mathrm{F}}=1.91 \times 10^{7} \mathrm{~cm} \mathrm{~s}^{-1}, \omega_{\mathrm{p}}=1.2 \mathrm{eV}$ and $H_{\mathrm{c} 2}(0)=49$ Tesla (Sparn et al 1991, 1992) to estimate the zero temperature elastic scattering rate of about $1.24 \times 10^{14} \mathrm{~s}^{-1}$ and is consistent as those derived from the superconducting fluctuation measurements (Xiang et al 1992). It is attributed to the fact that the larger the electron mass, the smaller the plasma frequency and hence the reduced zero temperature elastic scattering rate.

We have earlier estimated the zero temperature mean free path, $\ell$ of about $3.4 \mathrm{~nm}$ which is highly sensitive for carrier scattering. We further find that the product, $k_{\mathrm{F}} \ell$ $(\sim 17)$, seems to be much larger than unity indicates the metallic characteristics. It is worth to mention that the product, $\varepsilon_{\mathrm{F}} \tau \gg 1$, in the test material refers to the fact that the doped fullerides fall in the weak scattering limit. This is however, consistent with the $s$ wave superconductors. With these parameters, we estimate zero temperature limited resistivity $\left(\rho_{0}=2.4 \mathrm{~m} \Omega \mathrm{cm}\right)$ consistent with the single crystal result (Xiang et al 1992).

We complete our model calculations by analysing the temperature dependent resistivity for $\mathrm{K}_{3} \mathrm{C}_{60}$ superconductors.

\subsection{Normal state resistivity}

To formulate a specific model, we start with the general expression for the temperature dependent part of the resistivity (Grimvall 1981), given by

$$
\rho=\frac{3 \pi}{\hbar e^{2} v_{\mathrm{F}}^{2}} \int_{0}^{2 k_{\mathrm{F}}}|v(q)|^{2}\left\langle|S(q)|^{2}\right\rangle\left(\frac{1}{2 k_{\mathrm{F}}}\right)^{4} q^{3} \mathrm{~d} q .
$$

$v(q)$ is the Fourier transform of the potential associated with one lattice site and $S(q)$ being the structure factor. Following the Debye model it takes the following form

$$
|S(q)|^{2} \approx \frac{k_{\mathrm{B}} T}{M v_{\mathrm{s}}^{2}} f(x) .
$$

$f(x)$ being the statistical factor and is

$$
f(x)=x\left[e^{x}-1\right]^{-1}\left[1-e^{-x}\right]^{-1} .
$$

Thus the resistivity expression leads to

$$
\rho \approx\left(\frac{3}{\hbar e^{2} v_{\mathrm{F}}^{2}}\right) \frac{k_{\mathrm{B}} T}{M v_{\mathrm{S}}^{2}} \int_{0}^{2 k_{\mathrm{F}}}|v(q)|^{2}\left[\frac{x q^{3} \mathrm{~d} q}{\left(e^{x}-1\right)\left(1-e^{-x}\right)}\right] .
$$

$v_{\mathrm{s}}$ being the sound velocity. Equation (69) in terms of intermolecular phonon contribution yields the BlochGruneisen function of temperature dependence resistivity

$$
\rho_{\mathrm{er}}\left(T, \theta_{\mathrm{er}}\right)=4 A_{\mathrm{er}}\left(T / \theta_{\mathrm{er}}\right)^{4} \times T \int_{0}^{\theta_{\mathrm{er}} / T} x^{5}\left(e^{x}-1\right)^{-1}\left(1-e^{-x}\right)^{-1} \mathrm{~d} x
$$


where, $x=\hbar \omega / k_{\mathrm{B}} T$. $A_{\mathrm{er}}$ being a constant of proportionality defined as

$$
A_{\mathrm{er}} \cong\left(3 \pi^{2} e^{2} k_{\mathrm{B}}\right)\left[k_{\mathrm{F}}^{2} v_{\mathrm{s}}^{2} L \hbar v_{\mathrm{F}}^{2} M\right]^{-1} .
$$

In view of inelastic neutron scattering measurements, the phonon spectrum can be conveniently separated into two parts of phonon density of states (Prassides et al 1991; Pintschovius 1996). It is natural to choose a model phonon spectrum consisting of two parts: an intermolecular phonon frequency, $\omega_{\mathrm{er}}\left(\theta_{\mathrm{er}}\right)$, and an intramolecular phonon frequency, $\omega_{\text {ra }}\left(\theta_{\text {ra }}\right)$. If the Matthiessen rule is obeyed, the resistivity may be represented as a sum $\rho(T)=\rho_{0}+\rho_{\mathrm{e}-\mathrm{ph}}$ $(T)$, where $\rho_{0}$ is the residual resistivity that does not depend on temperature as described earlier. On the other hand, in case of the intramolecular phonon spectrum, $\rho_{\text {ra }}$ (T) may be described as follows

$$
\begin{aligned}
\rho_{\text {ra }}\left(T, \theta_{\text {ra }}\right)= & A_{\text {ra }} \theta_{\text {ra }}^{2} T^{-1}\left[\exp \left(\theta_{\text {ra }} / T\right)^{-1}\right]^{-1} \times \\
& {\left[1-\exp \left(-\theta_{\text {ra }} / T\right)\right]^{-1} . }
\end{aligned}
$$

$A_{\text {ra }}$ is defined analogously to (60). Finally, the phonon resistivity reads

$$
\rho_{\mathrm{e}-\mathrm{ph}}(T)=\rho_{\mathrm{er}}\left(T, \theta_{\mathrm{er}}\right)+\rho_{\mathrm{ra}}\left(T, \theta_{\mathrm{ra}}\right) .
$$

Henceforth, the total resistivity is now rewritten as

$$
\begin{aligned}
\rho\left(T, \theta_{\mathrm{er}}, \theta_{\mathrm{ra}}\right)= & \rho_{0}+\rho_{\mathrm{er}}\left(T, \theta_{\mathrm{er}}\right)+\rho_{\mathrm{ra}}\left(T, \theta_{\mathrm{ra}}\right) \\
= & \rho_{0}+4 A_{\mathrm{er}}\left(T / \theta_{\mathrm{er}}\right)^{4} T \times \\
& \int_{\mathrm{er}} / T \\
& \int_{0}^{5}\left(e^{x}-1\right)^{-1}\left(1-e^{-x}\right)^{-1} \mathrm{~d} x+A_{\mathrm{ra}} \theta_{\mathrm{ra}}^{2} T^{-1} \\
& {\left[\exp \left(\theta_{\mathrm{ra}} / T\right)^{-1}\right]^{-1}\left[1-\exp \left(-\theta_{\mathrm{ra}} / T\right)\right]^{-1} . }
\end{aligned}
$$

Though this is a purely phenomenological expression, it seems to provide a reasonable description of the available experimental data. Having discussed the model, we now proceed to analyse numerically the temperature dependent contribution in $\mathrm{K}_{3} \mathrm{C}_{60}$ superconductor.

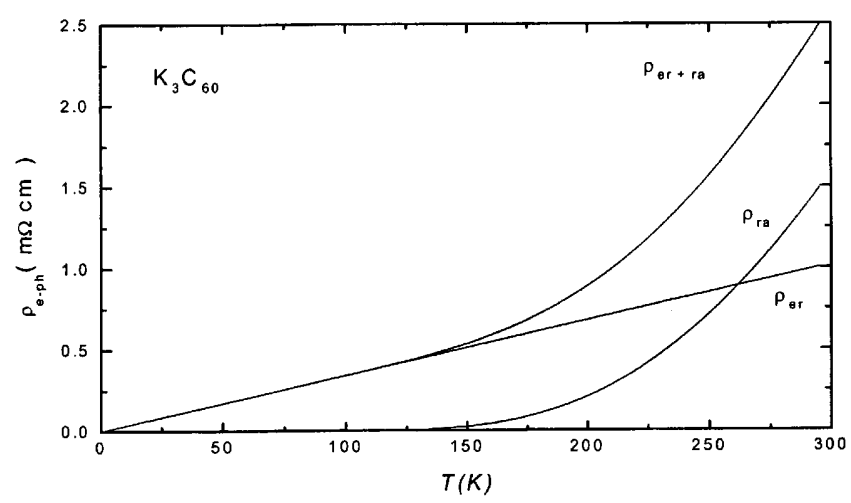

Figure 4. Variation of $\rho_{\mathrm{e}-\mathrm{ph}}$ with temperature, the contribution of $\rho_{\mathrm{er}}$ as well as $\rho_{\mathrm{ra}}$ to the resistivity.
Figure 4 illustrates the results of temperature dependence of resistivity via the ordinary electron-phonon interaction from (74) with our choice of intermolecular phonon frequency, $\omega_{\mathrm{er}}(=91 \mathrm{~K})$ and intramolecular phonon frequency, $\omega_{\mathrm{ra}}(=1455 \mathrm{~K})$. The contributions of intermolecular and intramolecular phonon towards resistivity are shown separately along with the total resistivity. It is inferred from the curve that $\rho_{\mathrm{er}}$ increases linearly, while $\rho_{\mathrm{ra}}$ increases exponentially with the increase in temperature. Both the contributions are clubbed together and the resultant resistivity is exponential at low temperatures, and nearly linear at high temperatures till room temperature.

Our numerical results on temperature dependence of resistivity of $\mathrm{K}_{3} \mathrm{C}_{60}$ are plotted in figure 5 along with the single crystal data (Xiang et al 1992). It is noticed from the plot that the estimated $\rho$ is lower than the reported data from $T_{\mathrm{c}}$ to near room temperature. Deduced values of the temperature dependent $\rho$ from (74) appears low, as $\rho_{0}$ and $\omega_{\mathrm{p}}$ values are the constraints for the present analysis. Thus estimated model parameters $\left[\lambda, \mu^{*}, v_{\mathrm{F}}, \omega_{\mathrm{p}}, \tau\right.$ and $\rho(0)]$ represent a good set of parameters for the estimation of normal state resistivity in alkali metal intercalated fullerides. Nevertheless, the role of electron-phonon interaction is better exploited and found prominent in the interpretation of normal state transport parameters.

The difference in between the measured $\rho$ and calculated $\rho_{\text {diff }}\left[=\rho_{\text {exp }}-\left\{\rho_{0}+\rho_{\mathrm{e}-\mathrm{ph}}\left(=\rho_{\mathrm{er}}+\rho_{\mathrm{ra}}\right)\right\}\right]$ is plotted in figure 6 . A power temperature dependence of $\rho_{\text {diff }}$ is inferred at low ( $\sim 22 \mathrm{~K}$ to $200 \mathrm{~K})$ temperature and it becomes almost saturate at higher temperature. The quadratic temperature contribution at low temperature for resistivity is an indication of conventional electron-electron scattering. The feature of quadratic temperature dependence of $\rho_{\text {diff }}$ is similar to that of electron doped cuprates, which is an artifact of electron-electron scattering (Varshney et al 2002). The departure from linear $T^{2}$ behaviour of $\rho_{\text {diff }}$ may be due to the dimensionality crossover if any.

Substantial deviation from the quadratic temperature dependence above $200 \mathrm{~K}$ may be due to possible struc-

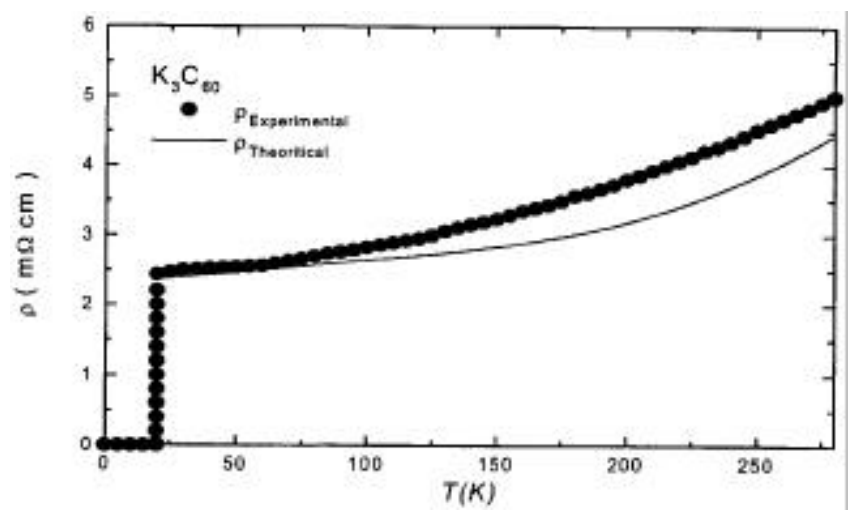

Figure 5. Variation of $\rho$ with temperature, $T(\mathrm{~K})$. Closed circles represent the experimental data taken from Xiang et al (1992). 


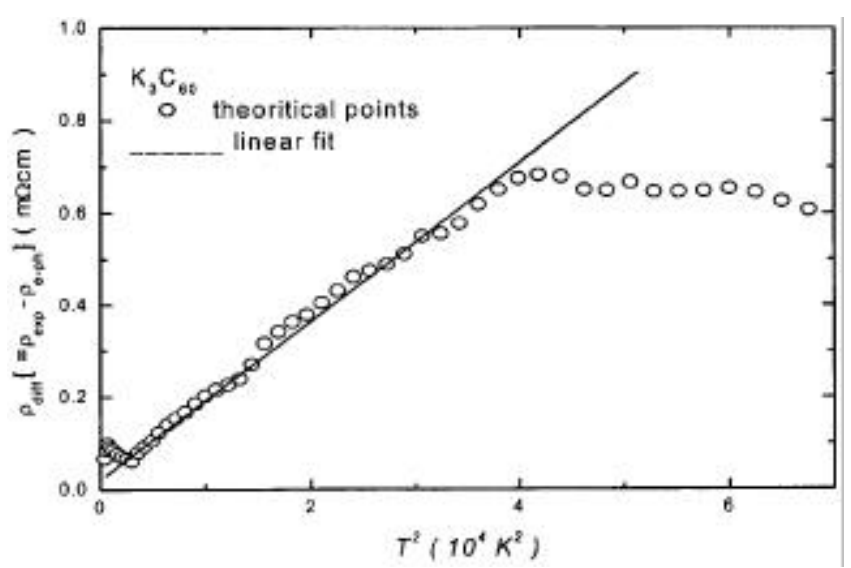

Figure 6. Variation of $\rho_{\text {diff }}\left[=\rho_{\text {exp }}-\left(\rho_{0}+\rho_{\mathrm{e}-\mathrm{ph}}\left\{\rho_{\mathrm{er}}+\rho_{\mathrm{ra}}\right\}\right)\right]$ (m $\Omega \mathrm{cm})$ with $T^{2}\left(10^{4} \mathrm{~K}^{2}\right)$.

tural changes but the exact reason is not clear. As $\mathrm{K}_{3} \mathrm{C}_{60}$ has a cubic structure, its electron system is not likely to change the dimensionality. Moreover, the transport properties may deviate from a Fermi liquid behaviour above $\sim 500 \mathrm{~K}$, suggesting a possible phase transition to a nonFermi liquid metallic state (Goldoni et al 2001) in thin film of $\mathrm{K}_{3} \mathrm{C}_{60}$. Therefore, more extensive theoretical and experimental determination of crossover temperature is required. The additional term due to electron-electron contribution was required in understanding the resistivity behaviour, as extensive attempts to fit the data with residual resistivity and phonon resistivity were unsuccessful. It is noteworthy to comment that in conventional metals, the electron-electron contribution to the resistivity can at best be seen at higher temperatures due to its small magnitude at low temperature when comparison is made with phonon contribution. We have thus demonstrated the role of electron-phonon scattering versus electron-electron interaction effects in determining the normal state resistivity of fullerides.

We refer to an earlier work of Thompson (1975), who postulated the power temperature dependence of electroresistivity in $\mathrm{TiS}_{2}$ as a consequence of low carrier concentration. Correlating this concept, alkali metal intercalated fullerenes has definitely low carrier concentration $\left(\sim 10^{21} \mathrm{~cm}^{-3}\right)$ and may result in a large enhancement of power temperature dependence of electrical resistivity even up to room temperature.

Analogously, one can shed further light by correlating the magnitude of electron-electron contribution to resistivity with plasma frequency. If the quadratic temperature dependence of resistivity is a cause of three-dimensional electron-electron scattering (umklapp), then its magnitude should depend on the carrier concentration $n$ as $n^{-5 / 3}$ or equivalently on the plasma frequency, as $\omega_{\mathrm{p}}^{-10 / 3}$. Within this scenario, we estimate $\rho_{\mathrm{ee}}$ of about $0.6 \mathrm{~m} \Omega \mathrm{cm}$ (as illustrated in figure 6) with $\omega_{\mathrm{P}}^{-10 / 3}$ as $0.54(\mathrm{eV})^{-10 / 3}$ for $\rho_{0}$ value of $2.4 \mathrm{~m} \Omega \mathrm{cm}$ in the vicinity of room temperature.
In conclusion, both qualitatively and quantitatively our results strongly suggest that all the three scales as Coulomb, intermolecular and intramolecular phonons are important in fullerides.

We note that Crespi et al (1992) have argued that electron-electron scattering mechanism is contributing to the normal state resistivity of $\mathrm{K}_{3} \mathrm{C}_{60}$ superconductors. They work with the Ziman's expression in analysing the single crystal $\mathrm{K}_{3} \mathrm{C}_{60}$ resistivity data. While interpreting the data they have used the value of coupling strength i.e. $\lambda_{\text {high }}$ and $\lambda_{\text {low }}$ as well as the molecular phonon frequencies from various theoretical models of the electron-phonon coupling and using $\alpha^{2} F(\omega)$ corresponding to a uniformly scaled version of the inelastic neutron scattering intensity. On the other hand in the present model calculations, we have used the coupling strength for electron-phonon coupling as deduced from the density of states, electronphonon interaction matrix and the inter-, intramolecular phonon frequency. The electron-ion matrix is derived from the Coulomb potential with static dielectric function in the long wavelength limit. Interestingly, the enhanced density of states adds the screening effect, which is properly incorporated in Coulomb potential.

We also refer to a recent photoemission spectra analysis of $\mathrm{K}_{3} \mathrm{C}_{60}$ which points to the metallicity of doped fullerides as a result of charge fluctuation occurring due to competition between the electron-electron as well as electron-phonon interaction (Chida et al 2002). However, there are several reports, which emphasize the importance of including both interactions in the understanding of fullerides (Knufper and Fink 1997). Thus, one cannot escape in stating that the temperature dependence of normal state resistivity in alkali metal doped fulleride is better understood by three-component model as $\rho=\rho_{\mathrm{o}}+\rho_{\mathrm{e}-\mathrm{ph}}+\rho_{\mathrm{e}-\mathrm{e}}$. Hence the physical entities leading to superconductivity and normal state electrical resistivity in doped fulleride can be better explained with the energy scales, alkali- $\mathrm{C}_{60}$ optic $\omega_{\mathrm{er}}$ (intermolecular vibrations), on ball- $\mathrm{C}_{60} \omega_{\mathrm{ra}}$ (intermolecular vibrations), and $\omega_{\mathrm{c}}$ (the Coulomb interactions).

\section{Conclusions}

In this paper, we have devoted our efforts in revealing the attractive pairing mechanism and various physical parameters of alkali metal intercalated fullerenes superconductor within the framework of a two-peak model for the phonon density of states. Considering the three dimensional $\mathrm{K}_{3} \mathrm{C}_{60}$ as a diatomic lattice of $\mathrm{K}$ and $\mathrm{C}_{60}$, we derive expression for intermolecular phonon modes. Looking to the importance of experimental constraints on high temperature superconducting theories, we have properly used the bulk modulus as well as other structural parameters. The approach takes care of structure for $8 \mathrm{~N} \mathrm{C}_{60}-\mathrm{K}$ bonds out of which $4 \mathrm{~N}$ are at octahedral position and $4 \mathrm{~N}$ takes 
the tetrahedral sites. We exercised the physical entities describing superconducting state and normal state electrical resistivity and presented our results on fullerides in terms of $s$ wave pairing state.

We properly use the RPA form of polarizability to obtain the electronic parameters as effective coupling strength. RPA is a valid concept in fullerides as it describes efficient screening for intermediate values of $U / W(\sim 1.0$ $2 \cdot 0)$. In this respect, the Coulomb pseudopotential is an essential parameter for superconductivity in fullerides. Thus we use rather simplified expression for effective coupling strength to understand various physical properties in fullerides instead of complicated quantum mechanical and band structure calculations. The proposed analysis reveals the condition $\mu^{*}<\mu, \lambda_{\text {er }}\left(\lambda_{\text {ra }}\right)-\mu^{*}>0$ to account for high- $T_{\mathrm{c}}$ in doped fullerides from the dependence of $T_{\mathrm{c}}$ on coupling strength. We find that $T_{\mathrm{c}}$ strongly depends on the Coulomb repulsive parameter and is an essential concept for superconductivity.

Also, by using the scaling factor as $\omega_{\text {er }}$ while estimating the screening parameter, we correctly represent the retardation effects that are small. As the Fermi energy is larger at least an order of magnitude by intermolecular phonons, the retardation effects are expected to be very small. On the other hand the Fermi energy and intramolecular phonon frequency are of almost similar order and hence vertex correction becomes important because the Migdal theorem does not hold and the frequency dependence of the effective Coulomb interaction must be considered in fullerides. Although we have provided a simple phenomenological explanation of screening parameter in fullerides, there is clearly a need for a detailed theoretical understanding of vertex corrections and shall be covered in near future.

The alkali- $\mathrm{C}_{60}$ intermolecular phonon participates in the pairing mechanism although strongly coupled but with little contribution as noticed from the transition temperature result. Keeping in mind that intramolecular phonons although moderately coupled indeed do play a significant role, we have obtained a steep increase in $T_{\mathrm{c}}$. However, we do introduce the intramolecular phonons in an adhoc way, we therefore obtained an analytical result and do not claim to possess a rigorous expression for them. The result is both inter and intramolecular phonons will induce the state as well as essential for superconducting fulleride properties. The proposed approach also explains consistently a reduced value of $\alpha$ when comparison is made with the BCS limit.

The reduced value of $\alpha$ essentially points to the large Coulomb repulsion apart from large electron-phonon coupling strength. In addition, the model calculations reveal that the coherence length which, even if small, is much larger than inverse Fermi momentum. The zero temperature mean free path, $\ell$ is much smaller than the zero temperature coherence length. The small value of $\ell$ is mainly attributed to the disorder, which is present in all alkali metal intercalated fulleride samples, including the best available single crystals. In this situation i.e. $\ell \ll \xi_{0}$, fullerides are considered to be in the dirty limit.

The appropriateness of the model calculations with both Coulomb and molecular (inter and intra) phonons is further revealed from the pressure dependence of $\mathrm{d} T_{\mathrm{c}} / \mathrm{d} P$ that show the metallic behaviour. The variation of $T_{\mathrm{c}}$ with pressure depends on the variation of the Fermi level density of states, the Coulomb pseudopotential and the variation of the phonon frequency with volume. The pressure effect in fullerides is huge and negative, while to that volume derivative of $T_{\mathrm{c}}$ is positive. Usually, the compression increases the bandwidth and hence the $\varepsilon_{\mathrm{F}}$ resulted in the reduction of renormalized screening parameter, and hence the contribution to $\mathrm{d} T_{\mathrm{c}} / \mathrm{d} V$ proportional to $\mathrm{d} \mu{ }^{*} / \mathrm{d} V$ is negative. We notice that intermolecular phonon frequencies are influenced under compression modes and if superconductivity in the fullerenes arose from coupling to intermolecular phonons then this argument implies strong electron-phonon coupling. We end by stating that both $T_{\mathrm{c}}$ and its pressure (volume) dependence are dictated by the properties of Coulomb, inter and intramolecular vibrations.

Within the framework of Bloch Gruneisen theory, we find that the normal state resistivity of alkali metal doped fullerenes consistent with single crystal data for $\mathrm{K}_{3} \mathrm{C}_{60}$ superconductors. We have first estimated the zero temperature elastic scattering rate with the use of parameters $\left(\lambda, \mu^{*}, v_{\mathrm{F}}, \tau, \mathrm{H}_{\mathrm{c} 2}(0)\right.$ and $\left.\omega_{\mathrm{p}}\right)$ from the developed approach. The larger mean free path and product $k_{\mathrm{F}} \ell \gg 1$ favours the metallic conduction. Hence, the use of Bloch Gruneisen expression in estimating the electron-phonon contributions is appropriate. It is noticed that contribution from intermolecular phonon and intramolecular phonon together with the zero temperature-limited resistivity is smaller than the reported data on the single crystal.

In view of inelastic neutron scattering data, the phonon spectrum is conveniently separated into two parts, intermolecular and intramolecular phonons. The high-energy intramolecular phonon yields a large contribution to the resistivity. We note that the product $\varepsilon_{\mathrm{F}} \tau \gg 1$ in the test material refers to the fact that the doped fullerides fall in the weak scattering limit. When the subtracted data is plotted as a function of $T^{2}$, a clear straight line is depicted from $22 \mathrm{~K}$ to $200 \mathrm{~K}$ but it becomes saturate at further higher temperature. The observation of $T^{2}$ dependence points toward the electron-electron scattering and departure from $T^{2}$ behaviour is an outcome of dimensionality crossover if any in these alkali metal intercalated fullerides.

The additional term due to electron-electron contribution was required in understanding the resistivity behaviour, as extensive attempts to fit the data with residual resistivity and phonon resistivity were unsuccessful. The magnitude of the resistivity is high for a metallic system, indicating a small density of carriers participating in the electrical conduction. The zero temperature-limited resis- 
tivity is also a large fraction of the total resistivity at room temperature, which suggests a large amount of impurity scattering is present. The large residual scattering is also susceptible to the phonon drag and as a consequence it decreases even in the single crystals. This presence of strong elastic scattering of electrons due to disorder may play a role in reducing the temperature dependence due to scattering by phonons below the $T^{5}$ behaviour seen in good metals with small elastic scattering, or the power temperature behaviour may arise from electron-electron scattering in Fermi liquid.

Conclusively, within the two peak model for phonon density of states the high frequency intramolecular phonon modes play a dominating role in pairing mechanism with possibly some contribution from alkali- $\mathrm{C}_{60}$ phonon to describe most of the superconducting and normal state properties of doped fullerides. Physically, we envisage a scenario in which the Coulomb screening apart from electron-phonon within RPA is an essential concept for superconductivity. It is a must to mention that the electron-intramolecular phonon coupling strength as well as the Coulomb pseudopotential from the simple method instead of rigorous quantum mechanical and electronic structure calculations is viable to retrace some of the experimental facts for alkali metal doped fullerides. The possibility of the cooperative mechanism in ammonia doped fullerides will gain an additional insight.

\section{Acknowledgements}

One of the authors (DV) thanks DRDO, Delhi, for generous financial assistance. (KKC) acknowledges AICTE, Delhi, for financial support under "Carrier Award for young teachers scheme".

\section{References}

Alexandrov A A 2001 Eur. Phys. Lett. 5692

Alexandrov A S and Kabanov V V 1996 Phys. Rev. $B 543655$

Belosludov V R and Shpakov V P 1991 Mod. Phys. Lett. 61209

Bogoliubov N N, Tolmachev V V and Shirkov D D 1959 A new method in the theory of superconductivity (NewYork: Consultants Bureau)

Burk B, Crespi V H, Zettl A and Cohen M L 1994 Phys. Rev. Lett. 723706

Cappelluti E, Grimaldi C, Pietronero L and Strassler S 2000 Phys. Rev. Lett. 854771

Cappelluti E, Grimaldi C, Pietronero L, Strassler S and Ummarino G A 2001 Eur. Phys. J. B21 383

Carbotte J P 1990 Rev. Mod. Phys. 6211012

Chaban I A 2002 J. Supercond: Incorp. Nov. Mag. 15179

Chen C C and Lieber C M 1993 Science 259655

Chida T, Suzuki S and Nakao K 2002 J. Phys. Soc. Jpn 71525

Crespi V H and Cohen M L 1996 Phys. Rev. B53 56

Crespi V H, Hou J G, Xiang X D, Cohen M L and Zettl A 1992 Phys. Rev. B46 12064
DeGiorgi L, Wachter P, Gruner G, Huang S-M, Wiley J and Kaner R B 1992 Phys. Rev. Lett. 692987

DeGiorgi L, Nicol E J, Klein O, Gruner G, Wachter P, Huang S M, Wiley J and Kaner R B 1994 Phys. Rev. B49 7012

Diederich J, Gangopadhyay A K and Schilling J S 1996 Phys. Rev. B54 R9662

Dresselhaus M S, Dresselhaus G and Eklund P C 1996 Science of fullerenes and carbon nanotubes (New York: Academic Press)

Erwin S C and Pickett W E 1992 Phys. Rev. B46 14257

Forro L and Mihaly L 2001 Rep. Prog. Phys. 64649

Fuhrer S, Cherrey K, Zettl A and Cohen M L 1999 Phys. Rev. Lett. 83404

Gelfand M P and Lu J P 1992 Phys. Rev. B46 4367

Goldoni A, Sangaletti, Parmigiani F, Comelli G and Paolucci G 2001 Phys. Rev. Lett. 87076401

Grimvall G 1981 The electron-phonon interaction in metals (Netherlands: North Holland Publishing Company)

Gunnarson O 1997 Rev. Mod. Phys. 69575

Hebard F 1992 Physics Today 4526

Hebard A F, Rosseinsky M J, Haddon R C, Murphy D W, Glarum S H, Palstra T T M, Ramirez A P and Kortan A R 1991 Nature 350600

Holczer K, Klein O, Huang S-M, Kaner R B, Fu K-J, Whetten R L and Diedrich F N 1991 Science 252600

Hou J G, Crespi V H, Xiang X D, Vareka W A, Briceno G, Zettl A and Cohen M L 1993 Solid State Commun. 86643

Hou J G, Lu Li, Crespi V H, Xiang X D, Zettl A and Cohen M L 1995 Solid State Commun. 93973

Huffman D R 1991 Physics Today 4422

Ivanov V and Maruyama Y 1995 Physica C247 147

Kerkoud R, Auban-Senzier P, Jerome D, Lambert J M, Zahab A and Bernier P 1994 Europhys. Lett. 25379

Kiefl R F et al 1993 Phys. Rev. Lett. 703987

Knufper M and Fink J 1997 Phys. Rev. Lett. 792714

Koller D, Martin M C, Mihaly L, Mihaly G, Oszlanyi G, Baumgartner G and Forro L 1996 Phys. Rev. Lett. 774082

Kresin V Z 1987 Phys. Lett. A122 434

Kresin V Z 1992 Phys. Rev. B46 14833

Maraduddin A A, Montroll E W and Weiss G H 1963 Theory of lattice dynamics in the harmonic approximation, Solid state physics suppl-3 (NewYork: Academic Press)

Martins J L and Troullier N 1992 Phys. Rev. B46 1766

McMillan W L 1968 Phys. Rev. 167331

Mitch M G, Chase S J and Lannian J S 1992 Phys. Rev. Lett. 68 883

Novikov D L, Gubanov V A and Freeman A J 1992 Physica C191 399

Palstra T T M, Haddon R C, Hebard A F and Zaaren J 1992 Phys. Rev. Lett. 641054

Pintschovius L 1996 Rep. Prog. Phys. 59473

Prassides K, Tomkinson J, Christides C, Rosseinsky M J, Murphy D W and Haddon R C 1991 Nature 354462

Ramirez A P et al 1992 Phys. Rev. Lett. 681058

Satapthy S, Antropov V O, Anderson O K, Jepsen O, Gunnarson O and Liechtension I 1992 Phys. Rev. B46 1773

Schluter M, Lanoo M, Needels M, Baratt G A and Tomanek D 1992 Phys. Rev. Lett. 68526

Sparn G, Thompson J D, Whetten R L, Huang S M, Kaner R B, Diederich F, Gruner G and Holczer K 1991 Science 2521829 Sparn G, Thompson J D, Whetten R L, Huang S M, Kaner R B, 
Diederich F, Gruner G and Holczer K 1992 Phys. Rev. Lett. 681228

Tanigaki K, Ebbessen T W, Saito S, Mizuki J, Tsai J S, Kubo Y and Kurushima S 1991 Nature 352222

Thompson A H 1975 Phys. Rev. Lett. 351786

Tycko R, Dabbagh G, Rosseinsky M J, Murphy D W, Ramirez A P and Fleming R M 1992 Phys. Rev. Lett. 681912

Varma C M, Zaanen J and Raghavachari K 1991 Science 254 989

Varshney Dinesh 2000 J. Superconductivity 13171

Varshney Dinesh and Singh R K 1997 Physica C 282-287 1919
Varshney Dinesh, Varshney M, Singh R K and Mishra R 1998 Supercond. Sci. Technol. 111300

Varshney Dinesh, Choudhary K K and Singh R K 2002 Supercond. Sci. Technol. 151119

Xiang X D, Hou J G, Briceno G, Vareka W A, Mostovoy R, Zettl A, Crespi V H and Cohen M L 1992 Science 2561190

Xiang X D, Hou J G, Crespi V H, Zettl A and Cohen M L 1993 Nature 36154

Zhang M L and Guo H Y 1994 Physica C227 15

Zhang F C, Ogata M and Rice T M 1991a Phys. Rev. Lett. 67 3452

Zhang Z, Chen C C and Lieber C M 1991b Science 2541619 